\title{
On Fourth-order Difference Equations for Orthogonal Polynomials of a Discrete Variable: Derivation, Factorization and Solutions
}

\author{
M. FOUPOUAGNIGNI ${ }^{a, *}$, W. KOEPF ${ }^{a}$ and A. RONVEAUX ${ }^{b}$ \\ ${ }^{a}$ Department of Mathematics and Computer Science, University of Kassel, Heinrich-Plett \\ Straße 40, 34132 Kassel, Germany; ${ }^{\text {b }}$ Facultés Universitaires Notre Dame de la Paix, \\ B-5000 Namur, Belgium
}

(Received 25 September 2002; Revised 11 January 2003; In final form 23 January 2003)

\begin{abstract}
We derive and factorize the fourth-order difference equations satisfied by orthogonal polynomials obtained from some modifications of the recurrence coefficients of classical discrete orthogonal polynomials such as: the associated, the general co-recursive, co-recursive associated, co-dilated and the general co-modified classical orthogonal polynomials. Moreover, we find four linearly independent solutions of these fourth-order difference equations, and show how the results obtained for modified classical discrete orthogonal polynomials can be extended to modified semi-classical discrete orthogonal polynomials. Finally, we extend the validity of the results obtained for the associated classical discrete orthogonal polynomials with integer order of association from integers to reals.
\end{abstract}

Keywords: Classical and semi-classical orthogonal polynomials of a discrete variable; $\Delta$-Laguerre-Hahn class; Functions of the second kind; Perturbed orthogonal polynomials; Second- and fourth-order difference equations

MSC 2000: Primary 33C45; Secondary 13P05

\section{INTRODUCTION}

Let $\mathscr{U}$ be a regular linear functional [3] on the linear space $\mathscr{P}$ of polynomials with real coefficient and $\left(P_{n}\right)_{n}$ a sequence of monic polynomials, orthogonal with respect to $\mathscr{U}$, i.e.

(i) $P_{n}(x)=x^{n}+$ lower degree terms,

(ii) $\left\langle\mathscr{U}, P_{n} P_{m}\right\rangle=k_{n} \delta_{n, m}, k_{n} \neq 0, \quad n \in \mathbb{N}$,

where $\mathbb{N}=\{0,1, \ldots\}$ denotes the set of non-negative integers. Here, $\langle\cdot, \cdot\rangle$ means the duality bracket and $\delta_{n, m}$ the Kronecker symbol.

$\left(P_{n}\right)_{n}$ satisfies a three-term recurrence equation

$$
P_{n+1}(x)=\left(x-\beta_{n}\right) P_{n}(x)-\gamma_{n} P_{n-1}(x), \quad n \geq 0,
$$

\footnotetext{
*Corresponding author. Address: Department of Mathematics, Advanced School of Education, University of Yaounde I, C/O: P.O. Box 8422 Yaounde, Cameroon. E-mail: foupoua@mathematik.uni-kassel.de; foumama@yahoo.fr; foupoua@uycdc.uninet.cm
} 
with the initial conditions

$$
P_{-1}(x)=0, \quad P_{0}(x)=1,
$$

where $\beta_{n}$ and $\gamma_{n}$ are real numbers with $\gamma_{n} \neq 0, \forall n \in \mathbb{N}_{>0}$ and $\mathbb{N}_{>0}$ denotes the set $\mathbb{N}_{>0}=\{1,2, \ldots\}$.

When the polynomial sequence $\left(P_{n}\right)_{n}$ is classical discrete [28,29], i.e. orthogonal with respect to a positive weight function $\rho$ defined on the set $I=\{a, a+1, \ldots, b-1\}$ and satisfying the first-order difference equation (called Pearson-type difference equation):

$$
\Delta(\sigma \rho)=\tau \rho,
$$

with

$$
\left.x^{n} \sigma(x) \rho(x)\right|_{x=a} ^{x=b}=0, \quad \forall n \in \mathbb{N},
$$

each $P_{n}$ satisfies the difference equation

$$
\mathbb{L}_{n}(y(x))=\sigma(x) \Delta \nabla y(x)+\tau(x) \Delta y(x)+\lambda_{n} y(x)=0,
$$

where

$$
\lambda_{n}=-\frac{n}{2}\left((n-1) \sigma^{\prime \prime}+2 \tau^{\prime}\right) .
$$

$\sigma$ is a polynomial of degree at most two and $\tau$ a first degree polynomial; the operators $\Delta$ and $\nabla$ are forward and backward difference operators defined by

$$
\Delta P(x)=P(x+1)-P(x), \quad \nabla P(x)=P(x)-P(x-1) \quad \forall P \in \mathscr{P} .
$$

The previous difference equation written in terms of forward and backward operators can be rewritten in terms of the shift operators as

$$
\begin{aligned}
\mathbb{D}_{n}(y(x))= & \left(\mathscr{T} \mathbb{L}_{n}\right)(y(x))=\left((\sigma(x+1)+\tau(x+1)) \mathscr{T}^{2}-(2 \sigma(x+1)\right. \\
& \left.\left.+\tau(x+1)-\lambda_{n}\right) \mathscr{T}+\sigma(x+1) \llbracket\right) y(x)=0, \quad n \geq 0
\end{aligned}
$$

where $\mathscr{T}$ and $\llbracket$ are the shift and the identity operators defined, respectively, by

$$
\mathscr{T} P(x)=P(x+1), \quad \llbracket P(x)=P(x) \quad \forall P \in \mathscr{P} .
$$

The orthogonality condition (ii) reads as

$$
\sum_{s=a}^{b-1} \rho(s) P_{n}(s) P_{m}(s)=k_{n} \delta_{n, m}, \quad k_{n} \neq 0, \quad \forall n \in \mathbb{N} .
$$

The coefficients $\beta_{n}, \gamma_{n}$ and $\lambda_{n}$ are given in Refs. [28,29] for any family of classical discrete orthogonal polynomials and in Refs. [12,16,18,34] in the generic case. The classical discrete families are Hahn, Kravchuk, Meixner and Charlier orthogonal polynomials [28].

Some modification of the recurrence coefficients $\left(\beta_{n}\right)_{n}$ and $\left(\gamma_{n}\right)_{n}$ of the Eq. (1) lead to new families of orthogonal polynomials (see Refs. [23,24,33] and references therein) such as the associated, the general co-recursive, co-recursive associated, co-dilated and the general co-modified classical discrete orthogonal polynomials [23]. Each of these new families of orthogonal polynomials satisfy a common fourth-order linear homogeneous difference 
equation with polynomial coefficients of bounded degree. In general, they cannot satisfy a common second-order linear homogeneous difference equation with polynomial coefficients of bounded degree. Therefore, these new polynomials are not semi-classical but belong to the discrete Laguerre-Hahn class (see "Preliminaries and notations" section). Many works have been devoted to the derivation of these fourth-order difference equations. Their polynomial coefficients have been given explicitly in Refs. $[1,7,8,10,19,32,36]$ for the $r$ th associated classical discrete orthogonal polynomials.

In 1999, using symmetry properties inside the three-term recurrence relation, hypergeometric representation and symbolic computation, the coefficients of the fourthorder difference equation for the co-recursive associated Meixner and Charlier orthogonal polynomials were given [20].

Despite the fact that the coefficients of the fourth-order difference equation satisfied by the perturbed classical discrete orthogonal polynomials require heavy computations for being very large, we have succeeded in deriving and factorizing these fourth-order difference equations and also finding a basis of four linearly independent solutions of all the difference equations satisfied by perturbed systems of the classical discrete orthogonal polynomials considered. Moreover, we have given explicitly the coefficients of the fourth-order difference equation satisfied by the $r$ th associated classical discrete orthogonal polynomials in terms of the polynomials $\sigma$ and $\tau$ appearing in Eq. (3). Also, we have found interesting relations between the perturbed polynomials, the starting ones and the functions of the second kind (see the next section for the definition). Therefore, the results obtained in the framework of this paper are more general and complete the known results in this area. In fact, we deal not only with the derivation of the fourth-order difference equation for the associated and the corecursive associated classical discrete orthogonal polynomials but with the derivation, the factorization and the solution basis of the fourth-order difference equations satisfied by the orthogonal polynomials obtained from some modifications of the recurrence coefficients of classical discrete orthogonal polynomials as was done for the continuous case [9]. Some examples of these families are the $r$ th associated, the generalized co-recursive, the generalized co-dilated, the generalized co-recursive associated and the generalized co-modified classical discrete orthogonal polynomials.

In the second section, we recall definitions and known results needed for this work. The third section is devoted to the derivation and the factorization of the fourth-order difference equation. In the fourth section, we solve difference equations and represent the perturbed classical orthogonal polynomials in terms of solutions of second-order difference equations. In the fifth section, we first give hypergeometric representation of solutions of Eq. (5) and difference operators $\mathbb{F}^{(r)}, \mathbb{S}^{(r)}$ and $\mathbb{T}^{(r)}$ for the $r$ th associated Charlier and Meixner orthogonal polynomials; secondly, we extend the results obtained for the associated orthogonal polynomials with integer order of association from integers to reals. Finally, we show how the results obtained for modified classical discrete orthogonal polynomials can be extended to modified semi-classical discrete orthogonal polynomials (see the next section for the definition).

\section{PRELIMINARIES AND NOTATIONS}

In this section, we first define the semi-classical and the Laguerre-Hahn class of a given family of orthogonal polynomials of a discrete variable. Next, we present the families of 
associated, generalized co-recursive, generalized co-recursive associated, generalized co-dilated and generalized co-modified orthogonal polynomials, and give relations between the new sequences and the starting ones.

Each linear functional $\mathscr{U}$ generates a so-called Stieltjes function $S$ of $\mathscr{U}$ defined by

$$
S(z)=-\sum_{n \geq 0} \frac{\left\langle\mathscr{U}, x^{n}\right\rangle}{z^{n+1}}
$$

where $\left\langle\mathscr{U}, x^{n}\right\rangle$ are the moments of the functional $\mathscr{U}$. The linear functional $\mathscr{U}$ satisfies in general a simple functional equation living in $\mathscr{P}^{\prime}$, the dual space of $\mathscr{P}$. Appropriate definitions of $\Delta(\mathscr{U})$ and $P \mathscr{U}$, where $P$ is a polynomial that allows building a simple difference equation for the functional, which generalizes in some way the Pearson-type difference equation for the weight $\rho[7,10,13,34]$.

If the Stieltjes function $S(x)$ satisfies a first-order linear difference equation of the form

$$
\phi(x) S(x+1)=C(x) S(x)+D(x),
$$

where $\phi, C$ and $D$ are polynomials, the functional $\mathscr{U}$ satisfies in $\mathscr{P}^{\prime}$ a first-order difference equation with polynomial coefficients. In this case, the functional $\mathscr{U}$ and the corresponding orthogonal polynomial sequence $\left(P_{n}\right)_{n}$ belong to the discrete semi-classical class (and are therefore called semi-classical discrete) which includes the classical discrete families $[7,10,13,14,25,26,34]$.

Each semi-classical discrete orthogonal polynomials sequence $\left(P_{n}\right)_{n}$ satisfies a common second-order difference equation $[7,10,13,14,22,25,26,34]$

$$
\mathbb{M}_{n}(y(x))=\left(I_{2}(x, n) \mathscr{T}^{2}+I_{1}(x, n) \mathscr{T}+I_{0}(x, n) \llbracket\right) y(x)=0,
$$

where $I_{i}(x, n)$ are polynomials in $x$ of degree not depending on $n$. Notice that this secondorder difference equation for the semi-classical discrete orthogonal polynomials appears in Ref. [10] as $\mathscr{D}_{0, n}(y)=0$ (using Equations 3.16 and 3.20).

An important class, larger than the semi-classical discrete one, appears when the Stieltjes function satisfies a $\Delta$-Riccati difference equation $[7,10,13]$

$$
\phi(x+1) \Delta S(x)=G(x) S(x) S(x+1)+E(x) S(x)+F(x) S(x+1)+H(x),
$$

where $\phi \neq 0, G, E, F$ and $H$ are polynomials fulfilling a certain conditions (see Ref. [11], Eq. 15). The corresponding functional $\mathscr{U}$ satisfies then a complicated quadratic difference equation in $\mathscr{P}^{\prime} . \mathscr{U}$ and the corresponding orthogonal polynomial families are said to belong to the discrete Laguerre-Hahn class $[7,10,13]$, denoted as $\Delta$-Laguerre-Hahn class.

It is well known that any $\Delta$-Laguerre-Hahn orthogonal polynomial sequence satisfies a common fourth-order difference equation of the form $[7,10]$

$$
\left(J_{4}(x, n) \mathscr{T}^{4}+J_{3}(x, n) \mathscr{T}^{3}+J_{2}(x, n) \mathscr{T}^{2}+J_{1}(x, n) \mathscr{T}+J_{0}(x, n) \llbracket\right) y(x)=0,
$$

where $J_{i}(x, n)$ are polynomials of degree not depending on $n$.

Furthermore, it is known that many perturbations of the recurrence coefficients of any Laguerre-Hahn family generate orthogonal polynomials belonging to the Laguerre-Hahn class and, therefore, satisfy a fourth-order differential or difference equation [7,10,13,21,34]. 


\subsection{Perturbation of Recurrence Coefficients}

Now we consider a sequence of polynomials $\left(P_{n}\right)_{n}$, orthogonal with respect to a regular linear functional $\mathscr{U}$, satisfying Eq. (1). Orthogonal families we will deal with are the associated orthogonal polynomials and those obtained from finite modification of the recurrence coefficients in Eq. (1). Some examples of these families are:

\subsubsection{The Associated Orthogonal Polynomials $\left(P_{n}^{(r)}\right)_{n}$}

Given $r \in \mathbb{N}$, the $r$ th associated of the polynomials $\left(P_{n}\right)_{n}$, is a polynomial sequence denoted by $\left(P_{n}^{(r)}\right)_{n}$ and defined by the recurrence equation (1) in which $\beta_{n}$ and $\gamma_{n}$ are replaced by $\beta_{n+r}$ and $\gamma_{n+r}$, respectively

$$
P_{n+1}^{(r)}(x)=\left(x-\beta_{n+r}\right) P_{n}^{(r)}(x)-\gamma_{n+r} P_{n-1}^{(r)}(x), \quad n \geq 1
$$

with the initial conditions

$$
P_{-1}^{(r)}(x)=0, \quad P_{0}^{(r)}(x)=1 .
$$

The family $\left(P_{n}^{(r)}\right)_{n}$, thanks to Favard's theorem [6] (see also Ref. [3]), is orthogonal. It is related to the starting polynomials and its first associated by the relation [4]

$$
P_{n}^{(r)}(x)=\frac{P_{r-1}(x)}{\Gamma_{r-1}} P_{n+r-1}^{(1)}(x)-\frac{P_{r-2}^{(1)}(x)}{\Gamma_{r-1}} P_{n+r}(x), \quad n \geq 0, \quad r \geq 1,
$$

where the sequence $\left(\Gamma_{n}\right)_{n}$ is defined by

$$
\Gamma_{n}=\prod_{i=1}^{n} \gamma_{i}, \quad n \geq 1, \quad \Gamma_{0} \equiv 1
$$

\subsubsection{The Co-recursive $\left(P_{n}^{[\mu]}\right)_{n}$ and the Generalized Co-recursive Orthogonal Polynomials $\left(\boldsymbol{P}_{n}^{[k, \mu]}\right)_{n}$}

The co-recursive of the orthogonal polynomial $\left(P_{n}\right)_{n}$, denoted by $\left(P_{n}^{[\mu]}\right)_{n}$, was introduced for the first time by Chihara [2], as the family of polynomials generated by the recursion formula (1) in which $\beta_{0}$ is replaced by $\beta_{0}+\mu$ :

$$
P_{n+1}^{[\mu]}(x)=\left(x-\beta_{n}\right) P_{n}^{[\mu]}(x)-\gamma_{n} P_{n-1}^{[\mu]}(x), \quad n \geq 1,
$$

with the initial conditions

$$
P_{0}^{[\mu]}(x)=1, \quad P_{1}^{[\mu]}(x)=x-\beta_{0}-\mu,
$$

where $\mu$ denotes a real number.

This notion was extended to the generalized co-recursive orthogonal polynomials in Refs. [4,5,31] by modifying the sequence $\left(\beta_{n}\right)_{n}$ at the level $k$. This yields an orthogonal polynomial sequence denoted by $\left(P_{n}^{[k, \mu]}\right)_{n}$ and generated by the recursion formula

$$
P_{n+1}^{[k, \mu]}(x)=\left(x-\beta_{n}^{*}\right) P_{n}^{[k, \mu]}(x)-\gamma_{n} P_{n-1}^{[k, \mu]}(x), \quad n \geq 1,
$$


with the initial conditions

$$
P_{0}^{[k, \mu]}(x)=1, \quad P_{1}^{[k, \mu]}(x)=x-\beta_{0}^{*},
$$

where $\beta_{n}^{*}=\beta_{n}$ for $n \neq k$ and $\beta_{k}^{*}=\beta_{k}+\mu$.

The orthogonal polynomial sequence $\left(P_{n}^{[k, \mu]}\right)_{n}$ is related to $\left(P_{n}\right)_{n}$ and is associated by Ref. [23]

$$
\begin{aligned}
& P_{n}^{[k, \mu]}(x)=P_{n}(x)-\mu P_{k}(x) P_{n-(k+1)}^{(k+1)}(x), \quad n \geq k+1, \\
& P_{n}^{[k, \mu]}(x)=P_{n}(x), \quad n \leq k .
\end{aligned}
$$

Use of Eq. (12) transforms the previous equations in

$$
\begin{aligned}
& P_{n}^{[k, \mu]}(x)=-\frac{\mu P_{k}^{2}(x)}{\Gamma_{k}} P_{n-1}^{(1)}(x)+\left(1+\frac{\mu P_{k}(x) P_{k-1}^{(1)}}{\Gamma_{k}}\right) P_{n}(x), \quad n \geq k+1, \\
& P_{n}^{[k, \mu]}(x)=P_{n}(x), \quad n \leq k .
\end{aligned}
$$

Obviously, we have the relations $P_{n}^{[0, \mu]}=P_{n}^{[\mu]}$ and $P_{n}^{[0]}(x)=P_{n}$.

\subsubsection{The Co-recursive Associated $\left(P_{n}^{\{r, \mu\}}\right)_{n}$ and the Generalized Co-recursive Associated Orthogonal Polynomials $\left(\boldsymbol{P}_{n}^{\{r, k, \mu\}}\right)_{n}$}

The co-recursive associated as well as the generalized co-recursive associated of the orthogonal polynomial sequence $\left(P_{n}\right)_{n}$, denoted by $\left(P_{n}^{\{r, \mu\}}\right)_{n}$ and $\left(P_{n}^{\{r, k, \mu\}}\right)_{n}$, respectively, are, the co-recursive and the generalized co-recursive (with modification on $\beta_{k}$ ) of the associated $\left(P_{n}^{(r)}\right)_{n}$ of $\left(P_{n}\right)_{n}$, respectively. Thanks to Eq. (18), they are related with $\left(P_{n}\right)_{n}$ and is associated by

$$
P_{n}^{\{r, 0, \mu\}}=P_{n}^{\{r, \mu\}},
$$

and

$$
\begin{aligned}
& P_{n}^{\{r, k, \mu\}}(x)=P_{n}^{(r)}(x)-\mu P_{k}^{(r)}(x) P_{n-(k+1)}^{(r+k+1)}(x), \quad n \geq k+1, \\
& P_{n}^{\{r, k, \mu\}}(x)=P_{n}^{(r)}(x), \quad n \leq k .
\end{aligned}
$$

The generalized co-recursive associated orthogonal polynomials can also be expressed using Eqs. (12) and (20) by

$$
\begin{aligned}
P_{n}^{\{r, k, \mu\}}(x)= & \left(\frac{P_{r-1}(x)}{\Gamma_{r-1}}-\frac{\mu P_{k+r}(x) P_{k}^{(r)}(x)}{\Gamma_{r+k}}\right) P_{n+r-1}^{(1)}(x) \\
& -\left(\frac{P_{r-2}^{(1)}(x)}{\Gamma_{r-1}}-\frac{\mu P_{k+r-1}^{(1)}(x) P_{k}^{(r)}(x)}{\Gamma_{r+k}}\right) P_{n+r}(x), \quad n \geq k+1, \\
P_{n}^{\{r, k, \mu\}}(x)= & P_{n}^{(r)}(x), \quad n \leq k .
\end{aligned}
$$




\subsubsection{The Co-dilated $\left(\boldsymbol{P}_{n}^{|\lambda|}\right)_{n}$ and the Generalized Co-dilated Orthogonal Polynomials $\left(\boldsymbol{P}_{n}^{|k, \lambda|}\right)_{n}$}

The co-dilated of the orthogonal polynomial sequence $\left(P_{n}\right)_{n}$, denoted by $\left(P_{n}^{|\lambda|}\right)_{n}$, was introduced by Dini [4], as the family of polynomials generated by the recursion formula (1) in which $\gamma_{1}$, is replaced by $\lambda \gamma_{1}$, i.e.

$$
P_{n+1}^{|\lambda|}(x)=\left(x-\beta_{n}\right) P_{n}^{|\lambda|}(x)-\gamma_{n} P_{n-1}^{|\lambda|}(x), \quad n \geq 2
$$

with the initial conditions

$$
P_{0}^{|\lambda|}(x)=1, \quad P_{1}^{|\lambda|}(x)=x-\beta_{0}, \quad P_{2}^{|\lambda|}(x)=\left(x-\beta_{0}\right)\left(x-\beta_{1}\right)-\lambda \gamma_{1},
$$

where $\lambda$ is a non-zero real number.

This notion was extended to the generalized co-dilated polynomials in Refs. [5,31] by modifying the sequence $\left(\gamma_{n}\right)_{n}$ at the level $k$. This yields an orthogonal polynomial sequence denoted by $\left(P_{n}^{|k, \lambda|}\right)_{n}$ and generated by the recurrence equation

$$
P_{n+1}^{|k, \lambda|}(x)=\left(x-\beta_{n}\right) P_{n}^{|k, \lambda|}(x)-\gamma_{n}^{*} P_{n-1}^{|k, \lambda|}(x), \quad n \geq 1,
$$

with the initial conditions

$$
P_{0}^{|k, \lambda|}(x)=1, \quad P_{1}^{|k, \lambda|}(x)=x-\beta_{0},
$$

where $\gamma_{n}^{*}=\gamma_{n}$ for $n \neq k$ and $\gamma_{k}^{*}=\lambda \gamma_{k}$.

The orthogonal polynomial sequence $\left(P_{n}^{|k, \lambda|}\right)_{n}$ is related to $\left(P_{n}\right)_{n}$ and is associated by Ref. [23]

$$
\begin{aligned}
& P_{n}^{|k, \lambda|}(x)=P_{n}(x)+(1-\lambda) \gamma_{k} P_{k-1}(x) P_{n-(k+1)}^{(k+1)}(x), \quad n \geq k+1, \\
& P_{n}^{|k, \lambda|}(x)=P_{n}(x), \quad n \leq k .
\end{aligned}
$$

Use of Eq. (12) transforms the previous equation in

$$
\begin{aligned}
& P_{n}^{|k, \lambda|}(x)=\left(1-\frac{(1-\lambda) P_{k-1}(x) P_{k-1}^{(1)}}{\Gamma_{k-1}}\right) P_{n}(x)+\frac{(1-\lambda) P_{k-1}(x) P_{k}(x)}{\Gamma_{k-1}} P_{n-1}^{(1)}(x), n \geq k+1 \\
& P_{n}^{|k, \lambda|}(x)=P_{n}(x), \quad n \leq k
\end{aligned}
$$

For $k=1$ or $\lambda=1$, we have

$$
P_{n}^{|1, \lambda|}=P_{n}^{|\lambda|}, \quad P_{n}^{|k, 1|}=P_{n} .
$$

\subsubsection{The Generalized Co-modified Orthogonal Polynomials $\left(P_{n}^{[k, \mu, \lambda]}\right)_{n}$}

New families of orthogonal polynomials can also be generated by modifying at the same time the sequences $\left(\beta_{n}\right)_{n}$ and $\left(\gamma_{n}\right)_{n}$ at the levels $k$ and $k^{\prime}$, respectively. When $k=k^{\prime}$, the new family obtained [23], denoted by $\left(P_{n}^{[k, \mu, \lambda]}\right)_{n}$ is generated by the three-term recurrence relation

$$
P_{n+1}^{[k, \mu, \lambda]}(x)=\left(x-\beta_{n}^{*}\right) P_{n}^{[k, \mu, \lambda]}(x)-\gamma_{n}^{*} P_{n-1}^{[k, \mu, \lambda]}(x), \quad n \geq 1,
$$


with the initial conditions

$$
P_{0}^{[k, \mu, \lambda]}(x)=1, \quad P_{1}^{[k, \mu, \lambda]}(x)=x-\beta_{0}^{*},
$$

where $\beta_{n}^{*}=\beta_{n}, \gamma_{n}^{*}=\gamma_{n}$ for $n \neq k$ and $\beta_{k}^{*}=\beta_{k}+\mu, \gamma_{k}^{*}=\lambda \gamma_{k}$. This family is represented in terms of the starting polynomials and their associated by Ref. [23]

$$
\begin{aligned}
P_{n}^{[k, \mu, \lambda]}(x) & =P_{n}(x)+\left((1-\lambda) \gamma_{k} P_{k-1}(x)-\mu P_{k}(x)\right) P_{n-(k+1)}^{(k+1)}(x), \quad n \geq k+1, \\
P_{n}^{|k, \lambda|}(x) & =P_{n}(x), \quad n \leq k
\end{aligned}
$$

The latter relation can also be written as

$$
\begin{aligned}
P_{n}^{[k, \mu, \lambda]}(x)= & \left(1-\frac{(1-\lambda) P_{k-1}(x) P_{k-1}^{(1)}}{\Gamma_{k-1}}+\frac{\mu P_{k}(x) P_{k-1}^{(1)}(x)}{\Gamma_{k}}\right) P_{n}(x) \\
& +\left(\frac{(1-\lambda) P_{k-1}(x) P_{k}(x)}{\Gamma_{k-1}}-\frac{\mu P_{k}^{2}(x)}{\Gamma_{k}}\right) P_{n-1}^{(1)}(x), \quad n \geq k+1, \\
P_{n}^{[k, \lambda]}(x)= & P_{n}(x), \quad n \leq k .
\end{aligned}
$$

\subsection{Results on Classical Discrete Orthogonal Polynomials}

Next, we state the following lemmas which are essential for this work. The first one is due to Atakishiyev, Ronveaux and Wolf [1] but the representation with the shift operator given by Eq. (32) is taken from Ref. [10] (see also Ref. [32]).

LEMMA 1 [1] Given a classical discrete orthogonal polynomial sequence $\left(P_{n}\right)_{n}$ satisfying Eq. (5), the following relation holds

$$
\mathbb{D}_{n}^{*}\left(P_{n-1}^{(l)}(x)\right)=\left(\frac{\sigma^{\prime \prime}}{2}-\tau^{\prime}\right)\left(\left(2 \sigma_{(I)}+\tau_{(I)}-\lambda_{n}\right) \mathscr{T}-\left(2 \sigma_{(l)}+\tau_{(I)}\right) \llbracket\right) P_{n}(x),
$$

where the operator $\mathbb{D}_{n}^{*}$ is given by

$$
\mathbb{D}_{n}^{*}=\left(\sigma_{(I)}+\tau_{(l)}\right)\left(\sigma_{(2)} \mathscr{T}^{2}-\left(2 \sigma_{(l)}+\tau_{(l)}-\lambda_{n}\right) \mathscr{T}+(\sigma+\tau) \llbracket\right)
$$

and

$$
\sigma \equiv \sigma(x), \quad \tau \equiv \tau(x), \quad \sigma_{(1)} \equiv \sigma(x+1), \quad \tau_{(l)} \equiv \tau(x+1), \quad \sigma_{(2)} \equiv \sigma(x+2) .
$$

It should be noticed that $\mathbb{D}_{n}$ and $\mathbb{D}_{n}^{*}$ are related by

$$
\sigma_{(l)} \mathbb{D}_{n}^{*}(\rho y)=\rho(\sigma+\tau)\left(\sigma_{(I)}+\tau_{(I)}\right) \mathbb{D}_{n}(y), \quad \forall y,
$$

where $\rho$ is the weight function satisfying Eqs. (3) and (4).

LEMMA 2 [28]

1. Two linearly independent solutions of the difference equation

$$
\mathbb{L}_{n}(y(x))=\sigma(x) \Delta \nabla y(x)+\tau(x) \Delta y(x)+\lambda_{n} y(x)=0,
$$


are $P_{n}$ and $Q_{n}$, where $\left(P_{n}\right)_{n}$ is a polynomial sequence, orthogonal with respect to the weight function $\rho$ defined on the set $I=\{a, a+1, \ldots, b-1\}$, satisfying Eqs. (3) and (4). The constants $\lambda_{n}$ is given by

$$
\lambda_{n}=-\frac{n}{2}\left((n-1) \sigma^{\prime \prime}+2 \tau^{\prime}\right),
$$

while $Q_{n}$ is the function of the second kind, defined by

$$
Q_{n}(x)=\frac{1}{\rho(x)} \sum_{s=a}^{b-1} \frac{\rho(s) P_{n}(s)}{s-x}, \quad x \notin\{a, a+1, \ldots, b-1\}
$$

When $x=t \in\{a, a+1, \ldots, b-1\}$, then $Q_{n}(t)$ is defined by

$$
Q_{n}(t)=\frac{1}{\rho(t)} \sum_{a \leq s \leq b-1, s \neq t}^{b-1} \frac{\rho(s) P_{n}(s)}{s-t}
$$

2. The polynomials $P_{n}$ and the function $Q_{n}$ are two linearly independent solutions of the recurrence equation (1).

\section{FACTORIZATION OF FOURTH-ORDER DIFFERENCE OPERATORS}

Given $\left(P_{n}\right)_{n}$ a classical discrete orthogonal polynomial sequence, we consider in general all transformations which lead to new families of orthogonal polynomials denoted by $\left(\bar{P}_{n}\right)_{n}$ and are related to the starting sequence by

$$
\bar{P}_{n}(x)=A_{n}(x) P_{n+k-1}^{(1)}+B_{n}(x) P_{n+k}, \quad n \geq k^{\prime},
$$

where $A_{n}$ and $B_{n}$ are polynomials of degree not depending on $n$, and $k, k^{\prime} \in \mathbb{N}$. (Among these transformations are the associated orthogonal polynomials and those obtained from finite modification of the recurrence coefficients of Eq. (1). Some examples are listed in Subsection 2.1).

We have the following:

\section{THEOREM 1}

1. The orthogonal polynomials $\left(\bar{P}_{n}\right)_{n \geq k^{\prime}}$ satisfy a common fourth-order linear difference equation

$$
\mathbb{F}_{n}(y(x))=\left(J_{4}(x, n) \mathscr{T}^{4}+J_{3}(x, n) \mathscr{T}^{3}+J_{2}(x, n) \mathscr{T}^{2}+J_{l}(x, n) \mathscr{T}+J_{0}(x, n) \llbracket\right) y(x)=0,
$$

where the coefficients $J_{i}$ are polynomials in $x$, with degree not depending on $n$.

2. The operator $\mathbb{F}_{n}$ can be factored as product of two-second order linear difference operators $\mathbb{S}_{n}$ and $\mathbb{T}_{n}$ :

$$
X_{n} \mathbb{F}_{n}=\mathbb{S}_{n} \mathbb{T}_{n}, \quad n \geq k
$$

where $X_{n}$ is a polynomial of fixed degree, depending on $P_{r-1}, \sigma$ and $\tau$, and the coefficients in $\mathbb{S}_{n}$ and $\mathbb{T}_{n}$ are polynomials of degree not depending on $n$. 
Proof In the first step, we solve Eq. (38) in terms of $P_{n+k-1}^{(1)}$

$$
P_{n+k-1}^{(I)}(x)=\frac{\bar{P}_{n}(x)-B_{n}(x) P_{n+k}(x)}{A_{n}(x)}
$$

and substitute the previous relation in Eq. (32) in which $n$ is replaced by $n+k$. Then we use Eq. (5) (for $P_{n+k}$ ) to eliminate the term $\mathscr{T}^{2} P_{n+k}$ and get

$$
\mathbb{M}_{n+k}\left(\bar{P}_{n}\right)=b_{1} \mathscr{T} P_{n+k}+b_{0} P_{n+k}
$$

where $b_{i}$ are rational functions and $\mathbb{M}_{n+k}$ a second-order linear difference operator given in terms of operator $\mathbb{D}_{n+k}^{*}$ (see Eq. (32)) by

$$
\mathbb{M}_{n+k}(y)=A_{n}\left(\mathscr{T} A_{n}\right)\left(\mathscr{T}^{2} A_{n}\right) \mathbb{D}_{n+k}^{*}\left(\frac{y}{A_{n}}\right) .
$$

Next, we shift Eq. (42) and use again Eq. (5) to eliminate $\mathscr{T}^{2} P_{n+k}$, and get

$$
\mathscr{T} \mathbb{M}_{n+k}\left(\bar{P}_{n}\right)=c_{1} \mathscr{T} P_{n+k}+c_{0} P_{n+k}
$$

We reiterate the same process using the previous equation and get

$$
\mathscr{T}^{2} \mathbb{M}_{n+k}\left(\bar{P}_{n}\right)=d_{l} \mathscr{T} P_{n+k}+d_{0} P_{n+k},
$$

where $c_{i}$ and $\mathrm{d}_{\mathrm{i}}$ are again rational functions.

The fourth-order difference equation is given in determinantal form from Eqs. (42), (44) and (45)

$$
\mathbb{F}_{n}\left(\bar{P}_{n}\right)=\left|\begin{array}{ccc}
b_{1} & b_{0} & \mathbb{M}_{n+k}\left(\bar{P}_{n}\right) \\
c_{1} & c_{0} & \mathscr{T} \mathbb{M}_{n+k}\left(\bar{P}_{n}\right) \\
d_{1} & d_{0} & \mathscr{T}^{2} \mathbb{M}_{n+k}\left(\bar{P}_{n}\right)
\end{array}\right|=0
$$

The previous equation can be written as

$$
\mathbb{F}_{n}\left(\bar{P}_{n}\right)=e_{2} \mathscr{T}^{2} \mathbb{M}_{n+k}\left(\bar{P}_{n}\right)+e_{1} \mathscr{T} \mathbb{M}_{n+k}\left(\bar{P}_{n}\right)+e_{0} \mathbb{M}_{n+k}\left(\bar{P}_{n}\right)=\left[\mathbb{S}_{n} \mathbb{T}_{n}\right]\left(\bar{P}_{n}\right)=0
$$

where the second-order difference operators $\mathbb{S}_{n}$ and $\mathbb{T}_{n}$ are given by

$$
\mathbb{S}_{n}=e_{2} \mathscr{T}^{2}+e_{1} \mathscr{T}+e_{0} \rrbracket, \quad \mathbb{T}_{n}=\mathbb{M}_{n+k}
$$

We conclude the proof by noticing that after cancellation of the denominator in Eq. (46), the coefficients $e_{i}$ are polynomials of degree not depending on $n$.

We would like to mention that the factorization pointed out in the previous theorem (except the case of the first associated classical discrete orthogonal polynomials already treated in Ref. [1] (see, also Ref. [10], equation 4.16 for more details) seems to be a new results and has lots of applications as will be shown later.

In what follows, we will denote, respectively, by $\mathbb{F}_{n}^{(r)}, \mathbb{F}_{n}^{[k, \mu]}, \mathbb{F}_{n}^{\{r, k, \mu\}}, \mathbb{F}_{n}^{|k, \lambda|}$ and $\mathbb{F}_{n}^{[k, \mu, \lambda]}$ the fourth-order difference operators for the $r$ th associated, the generalized co-recursive, the generalized co-recursive associated, the generalized co-dilated, and the generalized co-modified orthogonal polynomials. 


\subsection{Some Consequences}

For the $r$ th associated classical discrete orthogonal polynomials $\left(P_{n}^{(r)}\right)_{n}$, we have used the previous theorem and the representation given in Eq. (12) to compute the operators $\mathbb{S}_{n}$ and $\mathbb{T}_{n}$ using Maple 8 [27].

PROPOSITION 1 The two difference operator factors of the fourth-order difference operator for the rth associated classical discrete orthogonal polynomials are

$$
\begin{aligned}
& \mathbb{S}_{n}^{(r)}=\sigma_{(2)} P_{r-l}(x+1)\left(\tau_{(I)}+\sigma_{(l)}\right)^{2}\left(2 \tau_{(I)}-\tau+\sigma_{(2)}\right)\left(-3 \sigma_{2}+3 \sigma_{1}-\sigma\right) \\
& \times\left(-2 \tau_{(1)}+8 \sigma_{(1)}+\zeta-6 \sigma_{(2)}-2 \sigma+2 \tau\right) \mathscr{T}^{2}+\left(-\sigma_{(2)}\left(8 \sigma_{(1)}-6 \sigma_{(2)}-3 \sigma\right)\right. \\
& \times\left(\tau_{(1)}+\sigma_{(1)}\right) \sigma_{(1)} \zeta\left(-\zeta-\lambda_{\mathrm{r}-1}-8 \sigma_{(1)}-3 \tau+2 \sigma+8 \sigma_{(2)}+4 \tau_{(1)}\right) P_{r-1}(x)-\sigma_{(2)} \\
& \times\left(8 \sigma_{(1)}-6 \sigma_{(2)}-3 \sigma\right)\left(\tau_{(1)}+\sigma_{(1)}\right)\left(6 \sigma_{(2)}^{2} \sigma_{(1)}+2 \sigma_{(2)} \tau_{(1)}^{2}-6 \sigma_{2} \sigma_{(1)} \tau_{(1)}-8 \sigma_{(2)} \sigma_{1}^{2}\right. \\
& +6 \sigma_{(2)}^{2} \tau_{(1)}+2 \sigma_{(2)} \sigma \tau_{(1)}-2 \sigma_{(2)} \tau \tau_{(1)}-2 \sigma_{(2)} \tau \sigma_{(1)}+2 \sigma_{(2)} \sigma_{(1)} \sigma+3 \tau \zeta \tau_{(1)}+6 \tau \zeta \sigma_{(1)} \\
& -3 \tau \zeta \lambda_{\mathrm{r}-1}-2 \sigma \zeta \tau_{(1)}-4 \sigma \zeta \sigma_{(1)}+2 \sigma \zeta \lambda_{\mathrm{r}-1}+5 \tau_{(1)} \lambda_{\mathrm{r}-1} \zeta-6 \lambda_{\mathrm{r}-1} \sigma_{(1)} \zeta-\zeta \lambda_{\mathrm{r}-1}^{2} \\
& +16 \zeta \sigma_{(1)}^{2}-4 \zeta \tau_{(1)}^{2}+\zeta^{2} \tau_{(1)}+2 \zeta^{2} \sigma_{(1)}-\zeta^{2} \lambda_{\mathrm{r}-1}+8 \lambda_{\mathrm{r}-1} \sigma_{(2)} \zeta-16 \sigma_{(2)} \sigma_{(1)} \zeta \\
& \left.\left.-8 \sigma_{(2)} \tau_{(1)} \zeta\right) P_{r-1}(x+1)\right) \mathscr{T}-\sigma_{(l)}\left(8 \sigma_{(I)}-6 \sigma_{(2)}-3 \sigma\right)\left(-3 \sigma_{(2)}+3 \sigma_{(l)}-\sigma\right) \\
& \times\left(2 P_{r-1}(x+1) \tau_{I}^{2}-3 P_{r-1}(x+1) \lambda_{r-1} \tau_{(I)}+\tau_{1} P_{r-1}(x+1) \sigma_{(2)}-\tau_{(I)} P_{r-1}(x+1) \tau\right. \\
& +4 \tau_{(1)} \sigma_{(1)} P_{r-l}(x+1)-2 \tau_{(l)} \sigma_{(l)} P_{r-l}(x)+3 \sigma_{(l)} P_{r-l}(x+1) \sigma_{(2)} \\
& -2 P_{r-1}(x+1) \sigma_{(2)} \lambda_{r-1}-2 P_{r-1}(x+1) \sigma_{(I)} \tau-2 \sigma_{(I)} \lambda_{r-1} P_{r-1}(x+1) \\
& +P_{r-1}(x+1) \lambda_{r-1} \tau+\lambda_{r-1}^{2} P_{r-1}(x+1)-2 \sigma_{l} \sigma_{(2)} P_{r-1}(x)+\sigma_{(I)} \lambda_{r-1} P_{r-1}(x) \\
& \left.+\sigma_{(l)} P_{r-l}(x)+\sigma_{(l)} P_{r-l}(x) \tau\right) \zeta \rrbracket, \\
& \mathbb{T}_{n}^{(r)}=\sigma_{(2)}^{2} P_{r-l}(x+1) P_{r-1}(x)\left(\tau_{(I)}+\sigma_{(I)}\right) \mathscr{T}^{2}-\sigma_{(2)} P_{r-l}(x)\left(-\tau_{(l)} P_{r-l}(x+1)\right. \\
& \left.\left.-2 \sigma_{(l)} P_{r-l}(x+1)+\sigma_{(l)} P r-l(x)+\lambda_{r-1} P_{r-l}(x+1)\right)\left(-2 \sigma_{(l)}+\lambda_{n+r}-\tau_{(l)}\right)\right) \mathscr{T} \\
& -\sigma_{(2)} P_{r-l}(x+1)(\sigma+\tau)\left(-\tau_{(I)} P_{r-l}(x+1)-2 \sigma_{(I) P r-l}(x+1)\right. \\
& \left.+\sigma_{(I)} P_{r-1}(x)+\lambda_{r-1} P_{r-1}(x+1)\right) \rrbracket,
\end{aligned}
$$

where $\left(P_{n}\right)_{n}$ is the sequence of classical orthogonal satisfying Eq. (5), $r \in \mathbb{N}_{>0}$ and

$$
\zeta=5 \tau_{(1)}-6 \sigma_{(1)}+8 \sigma_{(2)}+2 \sigma-3 \tau-\lambda_{r-1}-\lambda_{n+r} .
$$

Moreover, we have

$$
\mathbb{S}_{n}^{(r)} \mathbb{T}_{n}^{(r)}=X_{n}\left(\sigma, \tau, P_{r-1}, \lambda_{r-1}\right) \mathbb{F}_{n}^{(r)},
$$


where

$$
\begin{aligned}
X_{n} \equiv & X_{n}\left(\sigma, \tau, P_{r-1}, \lambda_{r-1}\right)=\sigma_{(2)} P_{r-1}(x+1)\left(-3 \sigma_{2}+3 \sigma_{(1)}-\sigma\right)\left(\tau_{(1)} P_{r-1}(x+1)\right. \\
& \left.+2 \sigma_{(1)} P_{r-1}(x+1)-\sigma_{(1)} P_{r-1}(x)-\lambda_{r-1} P_{r-1}(x+1)\right)\left(8 \sigma_{(1)}-6 \sigma_{(2)}-3 \sigma\right) \\
& \times\left(2 P_{r-1}(x+1) \tau_{(1)}^{2}-3 P_{r-1}(x+1) \lambda_{r-1} \tau_{1}+\tau_{(1)} P_{r-1}(x+1) \sigma_{(2)}-\tau_{(1)} P_{r-1}(x+1) \tau\right. \\
& +4 \tau_{(1)} \sigma_{(1)} P_{r-1}(x+1)-2 \tau_{(1)} \sigma_{(1)} P_{r-1}(x)+3 \sigma_{(1)} P_{r-1}(x+1) \sigma_{(2)}-2 P_{r-1}(x+1) \sigma_{(2)} \lambda_{r-1} \\
& -2 P_{r-1}(x+1) \sigma_{(1)} \tau-2 \sigma_{(1)} \lambda_{r-1} P_{r-1}(x+1)+P_{r-1}(x+1) \lambda_{r-1} \tau+\lambda_{r-1}^{2} P_{r-1}(x+1) \\
& \left.-2 \sigma_{(1)} \sigma_{(2)} P_{r-1}(x)+\sigma_{(1)} \lambda_{r-1} P_{r-1}(x)+\sigma_{(1)} P_{r-1}(x) \tau\right)
\end{aligned}
$$

and

$$
\mathbb{F}_{n}^{(r)}=I_{4}(x, n) \mathscr{T}^{4}+I_{3}(x, n) \mathscr{T}^{3}+I_{2}(x, n) \mathscr{T}^{2}+I_{1}(x, n) \mathscr{T}+I_{0}(x, n) \rrbracket,
$$

with

$$
I_{0}(x, n, r)=-\sigma_{(1)}(\sigma+\tau) \zeta,
$$

$$
\begin{aligned}
I_{1}(x, n, r)= & 6 \sigma_{(2)}^{2} \sigma_{1}+2 \sigma_{(2)} \tau_{(1)}^{2}-6 \sigma_{(2)} \sigma_{(1)} \tau_{(1)}-8 \sigma_{(2)} \sigma_{(1)}^{2}+6 \sigma_{(2)}^{2} \tau_{(1)}+2 \sigma_{(2)} \sigma \tau_{(1)} \\
& -2 \sigma_{(2)} \tau \tau_{(1)}-2 \sigma_{(2)} \tau \sigma_{(1)}+2 \sigma_{2} \sigma_{(1)} \sigma+3 \tau \zeta \tau_{(1)}+6 \tau \zeta \sigma_{(1)}-3 \tau \zeta \lambda_{r-1}-2 \sigma \zeta \tau_{(1)} \\
& -4 \sigma \zeta \sigma_{(1)}+2 \sigma \zeta \lambda_{r-1}+5 \tau_{(1)} \lambda_{r-1} \zeta-6 \lambda_{r-1} \sigma_{1} \zeta-\zeta \lambda_{r-1}^{2}+16 \zeta \sigma_{1}^{2}-4 \zeta \tau_{(1)}^{2}+\zeta^{2} \tau_{(1)} \\
& +2 \zeta^{2} \sigma_{(1)}-\zeta^{2} \lambda_{r-1}+8 \lambda_{r-1} \sigma_{(2)} \zeta-16 \sigma_{(2)} \sigma_{(1)} \zeta-8 \sigma_{(2)} \tau_{1} \zeta \\
I_{2}(x, n, r)= & -\zeta^{3}+\left(-5 \tau+14 \sigma_{(2)}-2 \lambda_{r-1}+4 \sigma+7 \tau_{1}-14 \sigma_{(1)}\right) \zeta^{2}+\left(-57 \sigma_{(2)}^{2}-4 \sigma^{2}\right. \\
& +12 \tau_{(1)} \lambda_{r-1}-20 \lambda_{r-1} \sigma_{(1)}-8 \tau^{2}+11 \sigma \tau+24 \tau \tau_{(1)}+41 \sigma_{(2)} \tau-37 \sigma_{(1)} \tau-8 \lambda_{r-1} \tau \\
& -16 \sigma \tau_{(1)}-31 \sigma_{(2)} \sigma+28 \sigma_{(1)} \sigma+6 \lambda_{r-1} \sigma-2 \lambda_{r-1}^{2}-48 \sigma_{(1)}^{2}-18 \tau_{(1)}^{2}+54 \sigma_{(1)} \tau_{(1)} \\
& \left.-59 \sigma_{(2)} \tau_{(1)}+22 \lambda_{r-1} \sigma_{(2)}+106 \sigma_{2} \sigma_{(1)}\right) \zeta+2\left(-4 \sigma_{(1)}-\tau+\sigma+3 \sigma_{(2)}\right. \\
& \left.+\tau_{(1)}\right)\left(3 \sigma_{(1)} \tau+6 \lambda_{r-1} \sigma_{(1)}-9 \sigma_{(2)} \sigma_{(1)}-6 \sigma_{(1)} \tau_{(1)}+2 \tau^{2}+3 \lambda_{r-1} \tau+3 \sigma_{(2)} \sigma\right. \\
& -7 \sigma_{(2)} \tau-\sigma \tau+2 \sigma \tau_{(1)}-7 \tau \tau_{(1)}-2 \lambda_{r-1} \sigma-8 \lambda_{r-1} \sigma_{(2)}+12 \sigma_{(2)} \tau_{(1)}-5 \tau_{(1)} \lambda_{r-1} \\
& \left.+9 \sigma_{(2)}^{2}+\lambda_{r-1}^{2}+6 \tau_{1}^{2}\right),
\end{aligned}
$$

$$
\begin{aligned}
I_{3}(x, n, r)= & \left(-2 \tau+6 \sigma_{(2)}-\lambda_{r-1}+2 \sigma+3 \tau_{(1)}-6 \sigma_{1}\right) \zeta^{2}-\left(-\lambda_{r-1}-8 \sigma_{(1)}-3 \tau+2 \sigma+8 \sigma_{(2)}\right. \\
& \left.+4 \tau_{(1)}\right)\left(-2 \tau+6 \sigma_{2}-\lambda_{r-1}+2 \sigma+3 \tau_{(1)}-6 \sigma_{(1)}\right) \zeta+2\left(-4 \sigma_{(1)}-\tau+\sigma+3 \sigma_{(2)}\right. \\
& \left.+\tau_{(1)}\right)\left(3 \sigma_{(1)} \tau+6 \lambda_{r-1} \sigma_{(1)}-9 \sigma_{(2)} \sigma_{(1)}-6 \sigma_{(1)} \tau_{(1)}+2 \tau^{2}+3 \lambda_{r-1} \tau+3 \sigma_{(2)} \sigma\right. \\
& -7 \sigma_{(2)} \tau-\sigma \tau+2 \sigma \tau_{1}-7 \tau \tau_{(1)}-2 \lambda_{r-1} \sigma-8 \lambda_{r-1} \sigma_{(2)}+12 \sigma_{(2)} \tau_{(1)}-5 \tau_{(1)} \lambda_{r-1} \\
& \left.+9 \sigma_{(2)}^{2}+\lambda_{r-1}^{2}+6 \tau_{(1)}^{2}\right)
\end{aligned}
$$




$$
\begin{aligned}
I_{4}(x, n, r)= & -\left(8 \sigma_{(1)}-6 \sigma_{(2)}-3 \sigma\right)\left(3 \sigma_{(2)}+\sigma-3 \sigma_{(1)}+3 \tau_{(1)}-2 \tau\right)\left(-8 \sigma_{(1)}+2 \sigma-2 \tau+2 \tau_{(1)}\right. \\
& \left.+6 \sigma_{(2)}-\zeta\right) .
\end{aligned}
$$

COROLLARY 1 The fourth-order difference operator can also be factorized as

$$
\tilde{\mathbb{S}}_{n}^{(r)} \tilde{\mathbb{T}}_{n}^{(r)}=X\left(\sigma, \tau, Q_{r-1}, \lambda_{r-1}\right) \mathbb{F}_{n}^{(r)},
$$

where the expression $X\left(\sigma, \tau, Q_{r-1}, \lambda_{r-1}\right)$ and the operators $\tilde{\mathbb{S}}_{n}^{(r)}$ and $\tilde{\mathbb{T}}_{n}^{(r)}$ are obtained from the expression $X\left(\sigma, \tau, P_{r-1}, \lambda_{r-1}\right)$ and operators $\mathbb{S}_{n}^{(r)}$ and $\mathbb{\mathbb { W }}_{n}^{(r)}$, respectively by replacing the polynomials $P_{r-1}$ with the function $Q_{r-1}$.

The proof is obtained by a direct computation using that $P_{n}$ and $Q_{n}$ satisfies Eq. (5).

PROPOSITION 2 The operator $\mathbb{T}_{n}$ for the generalized co-recursive and co-dilated classical discrete orthogonal polynomials $\left(P_{n}^{[k, \mu]}\right)_{n}$ and $\left(P_{n}^{|k, \lambda|}\right)_{n}$ (with $\left.k \geq 1\right)$, denoted, respectively by $\mathbb{T}_{n}^{[k, \mu]}, \mathbb{T}_{n}^{|k, \lambda|}$ are obtained in the same way:

$$
\begin{aligned}
& \mathbb{T}_{n}^{[k, \mu]}=\sigma_{(2)} P_{k}^{2}(x) P_{k}^{2}(x+1)\left(\tau_{(I)}+\sigma_{(I)}\right)^{2} \mathscr{T}^{2}-P_{k}^{2}(x)\left(\tau_{(I)}-\lambda_{n}+2 \sigma_{I}\right)\left(-\sigma_{(l)} P_{k}(x)\right. \\
& \left.+2 \sigma_{(l)} P_{k}(x+1)-\lambda_{k} P_{k}(x+1)+\tau_{(l)} P_{k}(x+1)\right)^{2} \mathscr{T}+P_{k}^{2}(x+1)(\sigma+\tau) \\
& \times\left(-\tau_{(I)} P_{k}(x+1)-2 \sigma_{(I)} P_{k}(x+1)+\sigma_{(I)} P_{k}(x)+\lambda_{k} P_{k}(x+1)\right)^{2} \rrbracket, \\
& \mathbb{T}_{n}^{|k, \lambda|}=\sigma_{(2)} P_{k-l}(x+1) P_{k}(x+1) P_{k-l}(x) P_{k}(x)\left(\tau_{(I)}+\sigma_{(I)}\right)^{2} \mathscr{T}^{2}-P_{k-l}(x) P_{k}(x) \\
& \times\left(\tau_{(I)}-\lambda_{n}+2 \sigma_{l}\right) \times\left(-\tau_{(l)} P_{k}(x+1)-2 \sigma_{(I)} P_{k}(x+1)+\sigma_{l} P_{k}(x)+\lambda_{k} P_{k}(x+1)\right) \\
& \times\left(-2 \sigma_{(l)} P_{k-l}(x+1)+\sigma_{(l)} P_{k-l}(x)-P_{k-l}(x+1) \tau_{(I)}+\lambda_{k-l} P_{k-l}(x+1)\right) \mathscr{T} \\
& +P_{k-1}(x+1) P_{k}(x+1)(\sigma+\tau)\left(-\tau_{(l)} P_{k}(x+1)-2 \sigma_{(l)} P_{k}(x+1)+\sigma_{(I)} P_{k}(x)\right. \\
& \left.+\lambda_{k} P_{k}(x+1)\right)\left(-2 \sigma_{(I)} P_{k-l}(x+1)+\sigma_{(I)} P_{k-l}(x)-P_{k-l}(x+1) \tau_{(I)}\right. \\
& \left.+\lambda_{k-1} P_{k-1}(x+1)\right) \rrbracket \text {. }
\end{aligned}
$$

The operators $\mathbb{S}_{n}$ for the generalized co-recursive and co-dilated classical orthogonal polynomials are very large expressions; however, they can be obtained using the previous theorem and Eqs. (21) and (31). The same remark applies for the factors $\mathbb{S}_{\mathrm{n}}$ and $\mathbb{T}_{\mathrm{n}}$ of the fourth-order difference equation satisfied by the generalized co-recursive associated and generalized co-modified classical orthogonal polynomials.

\section{SOLUTIONS OF THE FOURTH-ORDER DIFFERENCE EQUATIONS}

In the following, we solve the fourth-order difference equation satisfied by the five perturbations listed in the second section and represent the new families of orthogonal polynomials in terms of solutions of second-order difference equations.

THEOREM 2 Let $\left(P_{n}\right)_{n}$ be a classical discrete orthogonal polynomial sequence, $r \in \mathbb{N}_{>0}$ and $\left(P_{n}^{(r)}\right)_{n}$ the rth associated of $\left(P_{n}\right)_{n}$. Four linearly independent solutions of the difference 
equation

$$
\mathbb{F}_{n}^{(r)}(y)=0
$$

satisfied by $\left(P_{n}^{(r)}\right)_{n}$, where $\mathbb{F}_{n}^{(r)}$ is given by Eq. (52), are

$$
\begin{aligned}
& A_{n}^{(r)}(x)=\rho(x) P_{r-1}(x) P_{n+r}(x), \\
& \mathrm{B}_{\mathrm{n}}^{(\mathrm{r})}(\mathrm{x})=\rho(\mathrm{x}) P_{r-1}(x) Q_{n+r}(x), \\
& \mathrm{C}_{\mathrm{n}}^{(\mathrm{r})}(\mathrm{x})=\rho(\mathrm{x}) Q_{r-1}(x) P_{n+r}(x), \\
& D_{n}^{(r)}(x)=\rho(x) Q_{r-1}(x) Q_{n+r}(x),
\end{aligned}
$$

$Q_{n}$ denoting the function of second kind associated to $\left(P_{n}\right)_{n}$ which is defined by Eqs. (36) and (37).

Moreover, $P_{n}^{(r)}$ is related to these solutions by

$$
\begin{aligned}
P_{n}^{(r)}(x) & =\frac{B_{n}^{(r)}(x)-C_{n}^{(r)}(x)}{\gamma_{0} \Gamma_{\mathrm{r}-1}}=\frac{\rho(x)\left(P_{r-1}(x) Q_{n+r}(x)-Q_{r-1}(x) P_{n+r}(x)\right)}{\gamma_{0} \Gamma_{r-1}}, \quad \forall n \in \mathbb{N}, \\
\forall r & \in \mathbb{N}_{>0},
\end{aligned}
$$

where $\Gamma_{k}$ is given by Eq. (13) and $\gamma_{0}$ defined as

$$
\gamma_{0}=\sum_{s=a}^{b-1} \rho(s) .
$$

Proof In the first step, we solve the difference equation

$$
\mathbb{T}_{n}^{(r)}(y)=0
$$

To do this, we use Eqs. (12), (35), (38), (43) and (48) to get

$$
\begin{aligned}
\mathbb{T}_{n}^{(r)}(y) & =\mathbb{M}_{n+r}(y) \\
& =P_{r-1}(x) P_{r-1}(x+1) P_{r-1}(x+2) \mathbb{D}_{n+r}^{*}\left(\frac{y}{P_{r-1}}\right) \\
& =P_{r-1}(x) P_{r-1}(x+1) P_{r-1}(x+2) \rho(x)(\sigma(x)+\tau(x))\left(\sigma_{(l)}+\tau_{(l)}\right) \mathbb{D}_{n+r}(z) / \sigma_{(1)},
\end{aligned}
$$

where the functions $y$ and $z$ are related by $y=z \rho P_{r-1}$. Since the two linearly independent solutions of $\mathbb{D}_{n+r}(z)=0$ are $P_{n+r}$ and $Q_{n+r}$ (see Lemma 2), the two linearly independent solutions of $\mathbb{W}_{n}^{(r)}(y)=0$ (which are also solutions of Eq. (56) thanks to Eq. (51)) are

$$
A_{n}^{(r)}(x)=\rho(x) P_{r-1}(x) P_{n+r}(x), \quad B_{n}^{(r)}(x)=\rho(x) P_{r-1}(x) Q_{n+r}(x) .
$$

Use of Eqs. (50) and (53) taking care that the weight function $\rho$ and the function $Q_{n}$ satisfy Eqs. (3) and (5), respectively, leads to

$$
\tilde{\mathbb{T}}_{n}^{(r)}(y)=Q_{r-1}(x) Q_{r-1}(x+1) Q_{r-1}(x+2) \rho(x)(\sigma(x)+\tau(x))\left(\sigma_{(1)}+\tau_{(1)}\right) \mathbb{D}_{n+r}(z) / \sigma_{(1)},
$$

where the functions y and $\mathrm{z}$ are related by $y=z \rho Q_{r-1}$. Equation (62) permits us to conclude that the two independent solutions of $\tilde{\mathbb{T}}_{n}^{(r)}(y)=O$ (which are also solutions of Eq. (56) thanks 
to Eq. (53)) are given by

$$
C_{n}^{(r)}(x)=\rho(x) Q_{r-1}(x) Q_{n+r}(x), \quad D_{n}^{(r)}(x)=\rho(x) Q_{r-1}(x) Q_{n+r}(x) .
$$

The four solutions of Eq. (56) obtained are linearly independent since $P_{n}$ and $Q_{n}$ are two linearly independent solutions of Eq. (5) and have different asymptotic behavior (see Remark 1).

The proof of Eq. (58) already given in Ref. [9] uses the fact that since $\left(P_{n}\right)_{n}$ and $\left(Q_{n}\right)_{n}$ satisfy Eq. (1), each solution given in Eq. (57) satisfies the recurrence equation

$$
X_{n+1}=\left(x-\beta_{n+r}\right) X_{n}-\gamma_{n+r} X_{n-1}, \quad n \geq 1 .
$$

Remark 1 Following the method used in Ref. [28] (see p. 98), we get the asymptotic formula for $Q_{n}(\mathrm{z})$ in the discrete case

$$
Q_{n}(z)=-\frac{\prod_{i=0}^{n} \gamma_{i}}{\rho(z) z^{n+1}}\left(1+\mathrm{O}\left(\frac{1}{\mathrm{z}}\right)\right)
$$

provided that when $z \rightarrow \infty$, the shortest distance from $\mathrm{z}$ to $(\mathrm{a}, \mathrm{b})$ is bounded away from zero. The previous asymptotic formula can be used to deduce the asymptotic formula for the solutions of the fourth-order difference equation give in Eq. (57).

If we replace the function of second kind $Q_{n}$ in Eqs. (57) and (58) by $\bar{Q}_{n}$ such that $P_{n}$ and $\bar{Q}_{n}$ are two linearly independent solutions of Eq. (1) (with the initial condition $\bar{Q}_{-1}(x)=$ $-(1 / \rho(x))$ and $\bar{Q}_{0}(x)$ fixed) and Eq. (5), then the four linearly independent solutions of Eq. (56) are obtained just by replacing $Q_{n}$ in Eq. (57) by $\bar{Q}_{n}$. Also, the relation between $P_{n}^{(r)}$, $P_{n}$ and $\bar{Q}_{n}$ is obtained by replacing $Q_{n}$ in Eq. (58) by $\bar{Q}_{n}$; however, the denominator $\gamma_{0} \Gamma_{r}$ of Eq. (58) is to be replaced by the term $\rho(x)\left(P_{r-1}(x) \bar{Q}_{r}(x)-\bar{Q}_{r-1}(x) P_{r}(x)\right)$ which is constant with respect to $x$. This remark applies also for Theorems 3-6.

THEOREM 3 Let $\left(\mathrm{P}_{\mathrm{n}}\right)_{\mathrm{n}}$ be a classical discrete orthogonal polynomial sequence, $k \in \mathbb{N}$ and $\left(P_{n}^{[k, \mu]}\right)_{\mathrm{n}}$ the generalized co-recursive of $\left(P_{n}\right)_{n}$. Four linearly independent solutions of the difference equation

$$
\mathbb{F}_{n}^{[k, \mu]}(y)=0, \quad n \geq k+1,
$$

satisfied by $\left(P_{n}^{[k, \mu]}\right)_{n}$, are (with $n \geq k+1$ )

$$
\begin{aligned}
& A_{n}^{[k, \mu]}(x)=\rho(x) P_{k}^{2}(x) P_{n}(x), \\
& B_{n}^{[k, \mu]}(x)=\rho(x) P_{k}^{2}(x) Q_{n}(x), \\
& C_{n}^{[k, \mu]}(x)=\left[\gamma_{0} \Gamma_{\mathrm{k}}+\mu \rho(\mathrm{x}) P_{k}(x) Q_{k}(x)\right] P_{n}(x), \\
& D_{n}^{[k, \mu]}(x)=\left[\gamma_{0} \Gamma_{\mathrm{k}}+\mu \rho(\mathrm{x}) P_{k}(x) Q_{k}(x)\right] Q_{n}(x),
\end{aligned}
$$

where $Q_{n}$ is the function of second kind associated to $\left(P_{n}\right)_{n}$ defined by Eqs. (36) and (37). 
Moreover, $P_{n}^{[k, \mu]}$ is related to these solutions by

$$
P_{n}^{[k, \mu]}=\frac{\left[\gamma_{0} \Gamma_{k}+\mu \rho(x) P_{k}(x) \mathrm{Q}_{k}(x)\right] P_{n}(x)-\mu \rho(x) P_{k}^{2}(x) Q_{n}(x)}{\gamma_{0} \Gamma_{\mathrm{k}}}, \quad k \geq 0, \quad n \geq k+1 .
$$

Proof By analogy with the proof of Theorem 2, we show using Eqs. (19), (43) and (48) that

$$
\mathbb{\mathbb { T }}_{n}^{[k, \mu]}(y)=\rho(\sigma+\tau)\left(\sigma_{(I)}+\tau_{(I)}\right) P_{k}^{2}(x) P_{k}^{2}(x+1) P_{k}^{2}(x+2) \mathbb{D}_{n}(z) / \sigma_{(I)},
$$

where $\mathbb{T}_{n}^{[k, \mu]}$ is given by Eq. (50) and $y(x)=z(x) \rho(x) P_{k}^{2}(x)$. Therefore, $A_{n}^{[k, \mu]}$ and $B_{n}^{[k, \mu]}$ given by

$$
A_{n}^{[k, \mu]}(x)=\rho(x) P_{k}^{2}(x) P_{n}(x), \quad B_{n}^{[k, \mu]}(x)=\rho(x) P_{k}^{2}(x) Q_{n}(x),
$$

are two linearly independent solutions of

$$
\mathbb{T}_{n}^{[k, \mu]}(y)=0 .
$$

Next, straightforward computation using Eqs. (19), (58) and (65) leads to

$$
P_{n}^{[k, \mu]}=\frac{C_{n}^{[k, \mu]}-\mu B_{n}^{[k, \mu]}}{\gamma_{0} \Gamma_{\mathrm{k}}}, \quad n \geq k+1 .
$$

Since the generalized co-dilated polynomials $P_{n}^{[k, \mu]}$ and the function $B_{n}^{[k, \mu]}$ given by Eq. (65), are both solutions of the linear homogenous difference equation

$$
\mathbb{F}_{n}^{[k, \mu]}(y)=0, \quad n \geq k+1
$$

it follows from Eq. (67) that the function $C_{n}^{[k, \mu]}$, given by Eq. (65), is also a solution of the previous equation.

To prove that the function $D_{n}^{[k, \mu]}$ is solution of Eq. (68), we proceed as follows:

In the first step, we write the expression $\mathbb{F}_{n}^{[k, \mu]}\left(C_{n}^{[k, \mu]}\right)$ in terms of $P_{n}(x)$ and $P_{n}(x+1)$ using the first-order difference equation satisfied by the weight (see Eq. (3)) and the second-order difference equation satisfied by $P_{n}$ (see Eq. (5))

$$
\mathbb{F}_{n}^{[k, \mu]}\left(C_{n}^{[k, \mu]}(x)\right)=G_{n}^{[k, \mu]}(x) P_{n}(x)+H_{n}^{[k, \mu]}(x) P_{n}(x+1), \quad n \geq k+1,
$$

where $G_{n}^{[k, \mu]}$ and $H_{n}^{[k, \mu]}$ are functions depending on $\rho, \sigma, \tau, P_{k}, Q_{k}$ and $\lambda_{n}$.

In the second step, we use the fact that $C_{n}^{[k, \mu]}$ is a solution of Eq. (64) and also the fact that $P_{n}(x)$ and $P_{n}(x+1)$ are linearly independent to deduce that

$$
G_{n}^{[k, \mu]}=H_{n}^{[k, \mu]}=0, \quad \text { for } n \geq k+1 .
$$

In fact, assuming that $G_{n}^{[k, \mu]}(x) \neq 0$, we get:

$$
G_{n}^{[k, \mu]}(x) P_{n}(x)+H_{n}^{[k, \mu]}(x) P_{n}(x+1)=0 \Longrightarrow P_{n}(x)=-\frac{H_{n}^{[k, \mu]}(x)}{G_{n}^{[k, \mu]}(x)} P_{n}(x+1) .
$$

We deduce that $G_{n}^{[k, \mu]}(x)=-H_{n}^{[k, \mu]}(x)$ (since $P_{n}$ is a monic polynomial of degree $n$ ). We conclude that

$$
0=G_{n}^{[k, \mu]}(x) P_{n}(x)+H_{n}^{[k, \mu]}(x) P_{n}(x+1)=-G_{n}^{[k, \mu]}(x) \Delta\left(P_{n}\right), \quad n \geq k+1 .
$$


The previous equation gives a contradiction because $G_{n}^{[k, \mu]} \neq 0$ and $\Delta\left(\mathrm{P}_{\mathrm{n}}\right) \neq 0$ (since $\left(\Delta\left(P_{n}\right)\right)_{n}$ is orthogonal with respect to $\left.\sigma(x+1) \rho(x+1)[29]\right)$.

Finally, we use the fact that $C_{n}^{[k, \mu]}$ and $D_{n}^{[k, \mu]}$ are multiples of $P_{n}$ and $Q_{n}$, respectively, with the same multiplier factor namely $\gamma_{0} \Gamma_{k}+\mu \rho P_{k} Q_{k}$ (see Eq. (65)), and the fact that $P_{n}$ and $Q_{n}$, satisfy the same second-order difference equation (5) to get

$$
\mathbb{F}_{n}^{[k, \mu]}\left(D_{n}^{[k, \mu]}(x)\right)=G_{n}^{[k, \mu]}(x) Q_{n}(x)+H_{n}^{[k, \mu]}(x) Q_{n}(x+1)=0, \quad n \geq k+1 .
$$

Therefore, $D_{n}^{[k, \mu]}$ is also a solution of Eq. (64).

To complete the proof, we notice that $A_{n}^{[k, \mu]}, B_{n}^{[k, \mu]}, C_{n}^{[k, \mu]}$ and $D_{n}^{[k, \mu]}$ are four linearly independent solutions of $\mathbb{F}_{n}^{[k, \mu]}(y)=0$ since $P_{n}$ and $Q_{n}$ are two linearly independent solutions of Eq. (5) enjoying different asymptotic properties.

In the following, we give the equivalent of the previous theorem for the co-dilated classical discrete orthogonal polynomials. The proof is similar to the one of the previous theorem by using relations (26), (27), (43), (48) and (58).

THEOREM 4 Let $\left(P_{n}\right)_{\mathrm{n}}$ be a classical discrete orthogonal polynomial sequence, $k \in \mathbb{N}$ and $\left(P_{n}^{|k, \lambda|}\right)_{n}$ the generalized co-dilated of $\left(P_{n}\right)_{n}$. Four linearly independent solutions of the difference equation

$$
\mathbb{F}_{n}^{|k, \mu|}(y)=0, \quad n \geq k+1,
$$

satisfied by $\left(P_{n}^{|k, \lambda|}\right)_{n}$ are (with $\left.n \geq k+1\right)$

$$
\begin{aligned}
& A_{n}^{|k, \lambda|}(x)=\rho(x) P_{k-1}(x) P_{k}(x) P_{n}(x), \\
& B_{n}^{|k, \lambda|}(x)=\rho(x) P_{k-1}(x) P_{k}(x) Q_{n}(x), \\
& C_{n}^{|k, \lambda|}(x)=\left[\gamma_{0} \Gamma_{k}+(\lambda-1) \gamma_{\mathrm{k}} \rho(\mathrm{x}) P_{k-1}(x) Q_{k}(x)\right] P_{n}(x), \\
& D_{n}^{|k, \lambda|}(x)=\left[\gamma_{0} \Gamma_{k}+(\lambda-1) \gamma_{\mathrm{k}} \rho(\mathrm{x}) P_{k-1}(x) Q_{k}(x)\right] Q_{n}(x) .
\end{aligned}
$$

The co-dilated $P_{n}^{|k, \lambda|}$ is related to these solutions by

$$
\begin{aligned}
P_{n}^{|k, \lambda|}= & \frac{\left[\gamma_{0} \Gamma_{k}+(\lambda-1) \gamma_{k} \rho(x) P_{k-1}(x) Q_{k}(x)\right] P_{n}(x)-(\lambda-1) \gamma_{k} \rho(x) P_{k-1}(x) P_{k}(x) Q_{n}(x)}{\gamma_{0} \Gamma_{k}} \\
& n \geq k+1
\end{aligned}
$$

We furthermore, give the solutions for the generalized co-recursive associated and the generalized co-modified classical orthogonal polynomials. The proofs are similar to the previous ones.

THEOREM 5 Let $\left(P_{n}\right)_{n}$ be a classical discrete orthogonal polynomial sequence, $k \in \mathbb{N}$, $r \in \mathbb{N}_{>0}$ and $\left(P_{n}^{\{r, k, \mu\}}\right)_{n}$ the generalized co-recursive associated with $\left(P_{n}\right)_{n}$. Four linearly independent solutions of the difference equation

$$
\mathbb{F}_{n}^{\{r, k, \mu\}}(y)=0, \quad n \geq k+1,
$$


satisfied by $\left(P_{n}^{\{r, k, \mu\}}\right)_{n}$ are (with $\left.n \geq k+1\right)$

$$
\begin{aligned}
& A_{n}^{\{r, k, \mu\}}(x)=\left(\gamma_{0} \Gamma_{k+r} P_{r-1}(x)-\mu \rho(x) P_{k+r}(x)\left[P_{r-1}(x) Q_{k+r}(x)-Q_{r-1}(x) P_{k+r}(x)\right]\right) \rho(x) P_{n+r}(x), \\
& B_{n}^{\{r, k, \mu\}}(x)=\left(\gamma_{0} \Gamma_{k+r} P_{r-1}(x)-\mu \rho(x) P_{k+r}(x)\left[P_{\mathrm{r} 1}(x) Q_{k+r}(x)-Q_{r-1}(x) P_{k+r}(x)\right]\right) \rho(x) Q_{n+r}(x), \\
& C_{n}^{\{r, k, \mu\}}(x)=\left(\gamma_{0} \Gamma_{k+r} Q_{r-1}(x)-\mu \rho(x) Q_{k+r}(x)\left[P_{r-1}(x) Q_{k+r}(x)-Q_{r-1}(x) P_{k+r}(x)\right]\right) \rho(x) P_{n+r}(x), \\
& D_{n}^{\{r, k, \mu\}}(x)=\left(\gamma_{0} \Gamma_{k+r} Q_{r-1}(x)-\mu \rho(x) Q_{k+r}(x)\left[P_{r-1}(x) Q_{k+r}(x)-Q_{r-1}(x) P_{k+r}(x)\right]\right) \rho(x) Q_{n+r}(x) .
\end{aligned}
$$

Moreover, $P_{n}^{\{r, k, \mu\}}$ is related to these solutions by

$$
\begin{aligned}
& P_{n}^{\{r, k, \mu\}}=\left(\frac{P_{r-1}(x)}{\gamma_{0} \Gamma_{r-1}}-\frac{\mu \rho(x) P_{k+r}(x)\left[P_{r-1}(x) \mathrm{Q}_{k+r}(x)-Q_{r-1}(x) P_{k+r}(x)\right]}{\gamma_{0}^{2} \Gamma_{r-1} \Gamma_{k+r}}\right) \rho(x) \mathrm{Q}_{n+r}(x) \\
&-\left(\frac{Q_{r-1}(x)}{\gamma_{0} \Gamma_{r-1}}-\frac{\mu \rho(x) Q_{k+r}(x)\left[P_{r-1}(x) Q_{k+r}(x)-Q_{r-1}(x) P_{k+r}(x)\right]}{\gamma_{0}^{2} \Gamma_{r-1} \Gamma_{k+r}}\right) \rho(x) P_{n+r}(x), \\
& r \geq 1, \quad n \geq k+1 .
\end{aligned}
$$

THEOREM 6 Let $\left(P_{n}\right)_{n}$ be a classical orthogonal polynomial sequence, $k \in \mathbb{N}$, and $\left(P_{n}^{[k, \mu, \lambda]}\right)_{n}$ the generalized co-modified of $\left(P_{n}\right)_{n}$. Four linearly independent solutions of the difference equation

$$
\mathbb{F}_{n}^{[k, \mu, \lambda]}(y)=0, \quad n \geq k+1,
$$

satisfied by $\left(P_{n}^{[k, \mu, \lambda]}\right)_{n}$ are (with $\left.n \geq k+1\right)$

$$
\begin{aligned}
& A_{n}^{[k, \mu, \lambda]}(x)=\left[(\lambda-1) \gamma_{k} P_{k-1}(x) P_{k}(x)+\mu P_{k}^{2}(x)\right] \rho(x) P_{n}(x), \\
& B_{n}^{[k, \mu, \lambda]}(x)=\left[(\lambda-1) \gamma_{k} P_{k-1}(x) P_{k}(x)+\mu P_{k}^{2}(x)\right] \rho(x) Q_{n}(x), \\
& C_{n}^{[k, \mu, \lambda]}(x)=\left[\gamma_{0} \Gamma_{\mathrm{k}}+(\lambda-1) \gamma_{\mathrm{k}} \rho(x) P_{k-1}(x) Q_{k}(x)+\mu \rho(x) P_{k}(x) Q_{k}(x)\right] P_{n}(x), \\
& D_{n}^{[k, \mu, \lambda]}(x)=\left[\gamma_{0} \Gamma_{\mathrm{k}}+(\lambda-1) \gamma_{\mathrm{k}} \rho(x) P_{k-1}(x) Q_{k}(x)+\mu \rho(x) P_{k}(x) Q_{k}(x)\right] Q_{n}(x) .
\end{aligned}
$$

The co-dilated $P_{n}^{[k, \mu, \lambda]}$ is related to these solutions by

$$
\begin{aligned}
P_{n}^{[k, \mu, \lambda]}= & \left(1+\frac{(\lambda-1) \gamma_{k} \rho(x) P_{k-1}(x) Q_{k}(x)+\mu \rho(x) P_{k}(x) Q_{k}(x)}{\gamma_{0} \Gamma_{k}}\right) P_{n}(x) \\
& -\frac{(\lambda-1) \gamma_{k} \rho(x) P_{k-1}(x) P_{k}(x)+\mu \rho(x) P_{k}^{2}(x)}{\gamma_{0} \Gamma_{k}} Q_{n}(x), \quad n \geq k+1 .
\end{aligned}
$$

\section{APPLICATIONS}

\subsection{On the rth Associated Charlier and Meixner Polynomials}

For Charlier and Meixner polynomials, we give explicitly the operators $\mathbb{S}_{n}^{(r)}, \mathbb{\mathbb { T }}_{n}^{(r)}, \mathbb{F}_{n}^{(r)}$ and the coefficient $X\left(\sigma, \tau, P_{r-1}, \lambda_{r-1}\right)$. We also give the hypergeometric representation of the two 
linearly independent solutions of Eq. (5) from which the hypergeometric representation of the four solutions of the four-order difference equation $\mathbb{F}_{n}^{(r)}(y)=0$ can be deduced.

\subsubsection{The Charlier Case}

The data for the Charlier polynomials $c_{n}^{(a)}(x)$ (denoted in this paper by $C_{n}(x, a)$ ) involved in Eqs. (1)-(5) are [17]:

$$
\sigma(x)=x, \quad \tau(x)=a-x, \quad \lambda_{n}=n, \quad \rho(x)=\frac{a^{x}}{x !}, \quad x \in \mathbb{N}, \quad \beta_{n}=n+a, \quad \gamma_{n}=n a, \quad a>0 .
$$

The recurrence equation as well as the difference equation (see Eqs. (1) and (5)) satisfied by the Charlier polynomials are given, respectively, by

$$
\begin{gathered}
a C_{n+1}(x, a)=(n+a-x) C_{n}(x, a)-n C_{n-1}(x, a), n \geq 1, C_{-1}(x, a)=0, C_{0}(x, a)=1, \\
a C_{n}(x+1, a)+(n-x-a) C_{n}(x, a)+x C_{n}(x-1, a)=0
\end{gathered}
$$

The monic Charlier polynomial $P_{n}(x)$ is related to the Charlier polynomial by

$$
P_{n}(x)=(-a)^{n} C_{n}(x, a),
$$

and satisfies the following normalized recurrence equation (see Eq. (1))

$$
P_{n+1}(x)=(x-n-a) P_{n}(x)-a n P_{n-1}(x), n \geq 1, P_{-1}(x)=0, P_{0}(x)=1 .
$$

The hypergeometeric representation of two linearly independent solutions of the recurrence equations (76) and (77) are given by

$$
\begin{gathered}
C_{n}(x, a)={ }_{2} F_{0}\left(\begin{array}{c|c}
-n,-x & 1 \\
- & -\frac{1}{a}
\end{array}\right), \\
\bar{C}_{n}(x, a)=\frac{1}{(x+1)(n+1)}{ }_{2} F_{2}\left(\begin{array}{c}
1,1 \\
n+2, x+2
\end{array}\right) .
\end{gathered}
$$

\section{Remark 2}

The polynomial $C_{n}(x, a)$ given by Eq. (79) is the Charlier polynomial and satisfies therefore Eqs. (76) and (77).

The function $\bar{C}_{n}(x, a)$ given by Eq. (80) satisfies also Eqs. (76) and (77). This can be verified by using the command sumrecursion [15] which gives the recurrence equation for sums of hypergeometric type.

$C_{n}(x, a)$ and $\bar{C}_{n}(x, a)$ are linearly independent solutions of Eq. (76) because the Casorati determinant of these solutions of the second-order difference equation (76) given 
by $(\rho(x)$ here is the Charlier weight)

$$
\begin{aligned}
W_{n}(x, a) & =C_{n-1}(x, a) \bar{C}_{n}(x, a)-C_{n}(x, a) \bar{C}_{n-1}(x, a) \\
& =\Gamma(n) \Gamma(x+1) 2^{-1} a^{x+n-1} \\
& =\frac{\Gamma(n) a^{1-n}}{2 \rho(x)}
\end{aligned}
$$

is different from zero.

$C_{n}(x, a)$ and $\bar{C}_{n}(x, a)$ are linearly independent solutions of Eq. (77) because they remain unchanged when we permutate the role of $x$ and $n$, and the difference equation (77) is obtained from Eq. (76) by permutation of $x$ and $n$.

The difference operators are given by

$$
\begin{aligned}
\mathbb{F}^{(r)}= & a(n+2 \zeta)(x+4) \mathscr{T}^{4}+\left(-2 a x-4 \zeta-2 \zeta^{3}+2 n^{2}-6 a+6 \zeta^{2}-3 n \zeta^{2}-n^{2} \zeta\right. \\
& +7 n \zeta-2 n) \mathscr{T}^{3}+\left(2 a x-5 a_{n}+2 \zeta+4 \zeta^{3}-n^{2}-4 \zeta a x-10 \zeta a+n^{3}+4 a-6 \zeta^{2}\right. \\
& \left.+6 n \zeta^{2}+4 n^{2} \zeta-4 n \zeta-2 a x n\right) \mathscr{T}^{2}+\left(2 a x+2 \zeta-2 \zeta^{3}+4 a-3 n \zeta^{2}-n^{2} \zeta+n \zeta\right) \mathscr{T} \\
& +a(n-2+2 \zeta)(x+1) \rrbracket \\
\mathbb{S}^{(r)}= & -a^{2}(x+2) P_{r-1}(x+1)(-n-2 \zeta)(x+3) \mathscr{T}^{2}+(-(x+2)(x+4)(n-2+2 \zeta) \\
& \times(n+\zeta+1)(x+1) P_{r-1}(x)+(x+2)(x+4)\left(2 a x+2 \zeta-2 \zeta^{3}+4 a-3 n \zeta^{2}-n^{2} \zeta+n \zeta\right) \\
& \left.\times P_{r-1}(x+1)\right) \mathscr{T}^{2}+a^{2}(x+2)(n+2 \zeta)(x+3) P_{r-1}(x+1) \rrbracket, \\
\mathbb{T}^{(r)}= & P_{r-1}(x+1) P_{r-1}(x)(x+2)^{2} a \mathscr{T}^{2}+\left(-(x+1)(n+\zeta+1)(x+2) P_{r-1}(x)^{2}-\zeta\right. \\
& \left.\times(n+\zeta+1)(x+2) P_{r-1}(x+1) P_{r-1}(x)\right) \mathscr{T}+\left(-a(x+1)(x+2) P_{r-1}(x+1) P_{r-1}(x)\right. \\
& \left.-\zeta a(x+2) P_{r-1}(x+1)^{2}\right) \rrbracket .
\end{aligned}
$$

Here, $\zeta$ is given by

$$
\zeta=r-x-a-2
$$

and $P_{r-1}$ represents the monic Charlier polynomial of degree $r-1$. The factor $X_{n}$ is given by

$$
\begin{aligned}
X_{n}\left(\sigma, \tau, P_{r-1}, \lambda_{r-1}\right)= & -(x+2)(x+3)(x+1)^{2}(x+4)(\zeta-1) P_{r-1}(x+1) P_{r-1}^{2}(x)+(x+4) \\
& \times(x+3)(x+2)(x+1)\left(a x+2 \zeta+2 a-2 \zeta^{2}\right) P_{r-1}^{2}(x+1) P_{r-1}(x) \\
& +(x+2)(x+3) \zeta(x+4)\left(\zeta+2 a+a x-\zeta^{2}\right) P_{r-1}^{3}(x+1) .
\end{aligned}
$$




\subsubsection{The Monic Meixner Case}

The data for the Meixner polynomials $m_{n}^{(b, c)}(x)$ (denoted in this paper by $M_{n}(x, b, c)$ ) are [17]:

$$
\begin{gathered}
\sigma(x)=x, \quad \tau(x)=(c-1) x+b c, \quad \lambda_{n}=(1-c) n, \quad \rho(x)=\frac{(b)_{x} c^{x}}{x !}, \quad x \in \mathbb{N}, \\
\beta_{n}=\frac{n+(n+b) c}{1-c}, \quad \gamma_{n}=\frac{n(n+b-1) c}{(1-c)^{2}}, \quad b>0, \quad 0<c<1,
\end{gathered}
$$

where $(b)_{x}$ represents the Pochhammer symbol defined by

$$
(b)_{x}=b(b+1) \ldots(b+x-1), \quad x \in \mathbb{N},(b)_{0} \equiv 1 .
$$

The recurrence equation as well as the difference equation (see Eqs. (1) and (5)) satisfied by the Meixner polynomials are given, respectively, by

$$
\begin{aligned}
& c(n+b) M_{n+1}(x, b, c)=(n(c+1)+b c+(c-1) x) M_{n}(x, b, c)-n M_{n-1}(x, b, c), \quad n \geq 1, \\
& M_{-1}(x, b, c)=0, \quad M_{0}(x, b, c)=1, \\
& c(x+b) M_{n}(x+1, b, c)-((1+c) x+b c+n(c-1)) M_{n}(x, b, c)+x M_{n}(x-1, b, c)=0 .
\end{aligned}
$$

The monic Meixner polynomial $P_{n}(x)$ is related to the Meixner polynomial $M_{n}(x, b, c)$ by

$$
P_{n}(x)=(b)_{n}\left(\frac{c}{c-1}\right)^{n} M_{n}(x, b, c),
$$

and satisfies the following normalized recurrence equation (see Eq. (1))

$$
\begin{aligned}
& P_{n+1}(x)=\left(x-\frac{n+(n+b) c}{1-c}\right) P_{n}(x)-\frac{n(n+b-1) c}{(1-c)^{2}} P_{n-1}(x), \\
& n \geq 1, P_{-1}(x)=0, P_{0}(x)=1 .
\end{aligned}
$$

Hypergeometric representations of two linearly independent solutions of Eqs. (81) and (82) are (with $b \neq 1$ )

$$
\begin{aligned}
& M_{n}(x, b, c)={ }_{2} F_{1}\left(\begin{array}{c}
-n,-x \\
b
\end{array} \mid 1-\frac{1}{c}\right), \\
& \bar{M}_{n}(x, b, c)=\frac{\Gamma(x+n+b+1) \Gamma(b)}{b(x+1)(n+1) \Gamma(x+b) \Gamma(n+b)}{ }_{3} F_{2}\left(\begin{array}{c}
1,1, x+n+b+1 \\
x+2, n+2
\end{array}\right) .
\end{aligned}
$$

Remark 3 The proof of the fact that $M_{n}(x, b, c)$ and $\bar{M}_{n}(x, b, c)$ are linearly independent solutions of Eqs. (81) and (82) (for $b \neq 1$ ) is obtained following the way indicated in Remark 2. In this case, the Casorati determinant of the solutions $M_{n}(x, b, c)$ and $\bar{M}_{n}(x, b, c)$ 
given by

$$
\begin{aligned}
Z_{n}(x, b, c) & =M_{n-1}(x, b, c) \bar{M}_{n}(x, b, c)-M_{n}(x, b, c) \bar{M}_{n-1}(x, b, c) \\
& =\frac{c^{1-x-n}(b-1) \Gamma(b)^{2} \Gamma(n) \Gamma(x+1)}{2 \Gamma(n+b) \Gamma(x+b)}=\frac{(b-1) \Gamma(n) c^{1-n}}{2(b)_{n} \rho(x)},
\end{aligned}
$$

where $\rho$ is the Meixner weight, vanishes only for $b=1$. Notice that the Casorati determinants $W_{n}(x, a)$ for Charlier and $Z_{n}(x, b, c)$ for Meixner cases were computed using the relations they satisfy

$$
W_{n+l}(x, a)=\frac{n}{a} W_{n}(x, a), \quad Z_{n+l}(x, b, c)=\frac{n}{c(n+b)} Z_{n}(x, b, c),
$$

and the Maple command sumrecursion [15] in order to find the first-order difference equations satisfied by $W_{l}(x, a)$ and $Z_{l}(x, b, c)$.

The function $\bar{C}_{n}(x, a)$ given by Eq. (80) can also be derived from $\bar{M}_{n}(x, b, c)$ (see Eq. (84)) using the following relation linking the Charlier and Meixner polynomials [17]

$$
\bar{C}_{n}(x, a)=\lim _{b \rightarrow \infty} \bar{M}_{n}\left(x, b, \frac{a}{a+b}\right) .
$$

Remark 4 The second solutions $\bar{C}_{n}(x, a)$ and $\bar{M}_{n}(x, b, c)$ of Eqs. (76) and (77) (for Charlier) and Eqs. (81) and (82) (for Meixner) given, respectively, by Eqs. (80) and (84) seem to be new results. These hypergeometric representations are covergent and were obtained in the following way: First, we neglect the first $x+1$ terms in the expression of $Q_{n}(x)$ given by Eq. (36) and get

$$
\bar{Q}_{n}(x)=\frac{1}{\rho(x)} \sum_{s=x+1}^{\infty} \frac{\rho(s) P_{n}(s)}{s+x} .
$$

Then we use the Maple command sumtohyper [15] to get the hypergeometric representation of $\bar{Q}_{n}(x)$ for the Charlier and Meixner polynomials. Finally, we remark that $\bar{Q}_{n}(x)$ satisfies Eq. (1) and multiply it by an appropriate factor in order to ensure the symmetry $\bar{Q}_{n}(x)=\bar{Q}_{x}(n)$.

The difference operators are given by

$$
\begin{aligned}
\mathbb{F}^{(r)}= & c(2 \zeta+N-c-1)(x+4)(b+x+3) \mathscr{T}^{4}-\left(4-2 b c x+2 c N^{2}+2 N^{2}-24 \zeta c-2 \zeta^{3}\right. \\
& -10 x c+9 \zeta^{2}+4 c^{3}+9 \zeta c N+9 \zeta N-\zeta N^{2}-12 \zeta c^{2}+9 \zeta^{2} c-3 \zeta^{2} N-2 x^{2} c^{2} \\
& \left.-2 b c^{2} x-10 x c^{2}-2 x^{2} c-6 b c^{2}-6 b c-6 N-6 c^{2} N-12 c N-12 \zeta\right) \mathscr{T}^{3} \\
& -\left(-2+4 b c x-8 x c N-5 b c N-4 c N^{2}-4 N^{2}+N^{3}+4 \zeta c+4 \zeta^{3}+14 x c-12 \zeta^{2}\right. \\
& -10 \zeta b c-4 \zeta x^{2} c-16 \zeta x c-4 \zeta b c x-2 c^{3}+6 c^{2}-12 \zeta c N-12 \zeta N+4 \zeta N^{2}+10 \zeta c^{2} \\
& -12 \zeta^{2} c+6 \zeta^{2} N+4 x^{2} c^{2}+4 b c^{2} x+14 x c^{2}+4 x^{2} c+9 b c^{2}+6 c+9 b c+5 N-2 N b c x \\
& \left.-2 N x^{2} c+5 c^{2} N+2 c N+10 \zeta\right) \mathscr{T}^{2}-\left(4 c^{2}+6 x c^{2}+2 x^{2} c^{2}+2 b c^{2} x+4 b c^{2}\right. \\
& +3 \zeta c N+3 \zeta^{2} c+4 c+6 x c+2 x^{2} c+2 b c x+4 b c+3 \zeta N+3 \zeta^{2}-3 \zeta^{2} N \\
& \left.-\zeta N^{2}-2 \zeta^{3}\right) \mathscr{T}+c(2 \zeta+N-3 c-3)(x+1)(b+x) \square,
\end{aligned}
$$




$$
\begin{aligned}
\mathbb{S}^{(r)}= & (x+2)(x+3)(1-2 \zeta+c-N)(b+x+2)(b+x+1)^{2} c^{3} P_{r-1}(x+1) \mathscr{T}^{2} \\
& -\left((x+2)(x+4) c(b+x+1)(x+1)(N+\zeta)(-N+3-2 \zeta+3 c) P_{r-1}(x)+(x+2)(x+4)\right. \\
& \times c(b+x+1)\left(4 c^{2}+6 x c^{2}+2 x^{2} c^{2}+2 b c^{2} x+4 b c^{2}+3 \zeta c N+3 \zeta^{2} c+4 c+6 x c+2 x^{2} c\right. \\
& \left.\left.+2 b c x+4 b c+3 \zeta N+3 \zeta^{2}-3 \zeta^{2} N-\zeta N^{2}-2 \zeta^{3}\right) P_{r-1}(x+1)\right) \mathscr{T}+(x+2)(x+3) \\
& \times(1-2 \zeta+c-N)(b+x+2)(b+x+1)^{2} c^{3} P_{r-1}(x+1) \rrbracket, \\
\mathbb{T}^{(r)}= & c(x+2)^{2}(b+x+1) P_{r-1}(x+1) P_{r-1}(x) \mathscr{T}^{2}+\left(-(x+2)(N+\zeta)(x+1) P_{r-1}(x)^{2}\right. \\
& \left.-\zeta(N+\zeta)(x+2) P_{r-1}(x+1) P_{r-1}(x)\right) \mathscr{T}+\left(-c(b+x)(x+1)(x+2) P_{r-1}(x+1) P_{r-1}(x)\right. \\
& \left.-(x+2) \zeta c(b+x) P_{r-1}(x+1)^{2}\right) \emptyset .
\end{aligned}
$$

Here $N$ and $\zeta$ are given by

$$
N=(n+1)(1-c), \quad \zeta=r-x-2-c(r+x+b)
$$

and $P_{r-1}$ is the monic Meixner polynomial of degree $r-1$. The expression $X_{n}$ in this case is given by

$$
\begin{aligned}
X_{n} \equiv & X_{n}\left(\sigma, \tau, P_{r-1}, \lambda_{r-1}\right) \\
= & (c-\zeta+1)(x+1)^{2}(x+2)(x+3)(x+4) P_{r-1}(x+1) P_{r-1}^{2}(x)+(x+1)(x+2)(x+3) \\
& \times(x+4)\left(x^{2} c+2 \zeta c+b c x+3 x c+2 c+2 b c+2 \zeta+2 \zeta^{2}\right) P_{r-1}^{2}(x+1) P_{r-1}(x)+\zeta(x+2) \\
& \times(x+3)(x+4)\left(x^{2} c+\zeta c+b c x+3 x c+2 c+2 b c+\zeta-\zeta^{2}\right) P_{r-1}^{3}(x+1) .
\end{aligned}
$$

Notice that the difference operators $\mathbb{F}_{n}^{(r)}$ given for the $r$ th associated Charlier and Meixner polynomials coincide with those given in Ref. [19] with the notations $\zeta=R, r=\gamma$.

\subsection{Extension of Results to Real Order of Association}

Let $\nu$ be a real number with $\nu \geq 0$ and $\left(P_{n}^{(r)}\right)_{n}$ the family of polynomials defined by

$$
P_{n+1}^{(\nu)}(x)=\left(x-\beta_{n+\nu}\right) P_{n}^{(\nu)}(x)-\gamma_{n+\nu} P_{n-1}^{(\nu)}(x), \quad n \geq 1
$$

with the initial conditions

$$
P_{0}^{(\nu)}(x)=1, \quad P_{1}^{(\nu)}(x)=x-\beta_{v},
$$

where $\beta_{n+\nu}$ and $\gamma_{n+\nu}$ are the coefficients $\beta_{n}$ and $\gamma_{n}$ of Eq. (1) with $n$ replaced by $n+\nu$.

We assume that the starting family $\left(P_{n}\right)_{n}$ defined in (1) is classical discrete. The coefficients $\beta_{n}$ and $\gamma_{n}$ are therefore rational function in the variable $n[16,18,28]$ and the coefficients $\beta_{n+\nu}$ and $\gamma_{n+\nu}$ well-defined. When $\gamma_{n+\nu} \neq 0, \forall n \geq 1$, the family $\left(P_{n}^{(r)}\right)_{n}$, thanks to Favard's theorem $[3,6]$ is orthogonal and represents the associated of the family $\left(P_{n}\right)_{n}$ with real order of association. 
THEOREM 7 Let $\left(P_{n}\right)_{n}$ be a family of classical discrete orthogonal polynomial, $\nu \geq 0$ a real number and $\left(P_{n}^{(\nu)}\right)_{n}$ the $\nu$-associated of $\left(P_{n}\right)_{n}$. We have:

1. $\left(P_{n}^{(\nu)}\right)_{n}$ satisfies

$$
\mathbb{F}_{n}^{(\nu)}(y)=0
$$

where $\mathbb{F}_{n}^{(\nu)}$ is the operator given in Eq. (52) with $r$ replaced by $\nu$.

2. The difference operator $\mathbb{F}_{n}^{(\nu)}$ factorizes as

$$
\mathbb{S}_{n}^{(\nu)} \mathbb{T}_{n}^{(\nu)}=X\left(\sigma, \tau, U_{\nu-1}, \lambda_{\nu-1}\right) \mathbb{F}_{n}^{(\nu)}, \quad \tilde{\mathbb{S}}_{n}^{(\nu)} \tilde{\mathbb{T}}_{n}^{(\nu)}=X\left(\sigma, \tau, V_{\nu-1}, \lambda_{\nu-1}\right) \mathbb{F}_{n}^{(\nu)},
$$

where the operators $\mathbb{S}_{n}^{(\nu)}, \mathbb{T}_{n}^{(\nu)}, \tilde{\mathbb{S}}_{n}^{(\nu)}, \tilde{\mathbb{T}}_{n}^{(\nu)}$ and the factor $X$ are those given in Eqs. (49)-(53) with $r$ replaced by $\nu, P_{r}$ and $Q_{r}$ are replaced by $U_{\nu}$ and $V_{\nu}$ respectively. $U_{\nu}$ and $V_{\nu}$ are the two linearly independent solutions of the difference equation (see Ref. [28,29])

$$
\sigma(x) \Delta \nabla y(x)+\tau(x) \Delta y(x)+\lambda_{\nu} y(x)=0,
$$

with $U_{r}=P_{r}, V_{r}=Q_{r}$ for $\nu=r \in \mathbb{N}$ and

$$
\lambda_{\nu}=-\frac{\nu}{2}\left((\nu-1) \sigma^{\prime \prime}+2 \tau^{\prime}\right)
$$

Four linearly independent solutions of difference equation (86) are given by

$$
\begin{aligned}
& A_{n}^{(\nu)}(x)=\rho(x) U_{\nu-1}(x) U_{n+\nu}(x), \\
& B_{n}^{(\nu)}(x)=\rho(x) U_{\nu-1}(x) V_{n+\nu}(x), \\
& C_{n}^{(\nu)}(x)=\rho(x) V_{\nu-1}(x) U_{n+\nu}(x), \\
& D_{n}^{(\nu)}(x)=\rho(x) V_{\nu-1}(x) V_{n+\nu}(x),
\end{aligned}
$$

where $\rho(x)$ is the weight function given by Eq. (3).

Proof

1. Let $n$ be a fixed integer number and define the function $\Phi$ by

$$
\Phi: \mathbb{R}_{+} \rightarrow \mathbb{R} \quad \nu \rightarrow \mathbb{F}_{n}^{(\nu)}\left(P_{n}^{(\nu)}(x)\right),
$$

where $\mathbb{R}_{+}$is the set of positive real numbers. Using relation (85) for fixed $x, \Phi(\nu)$ can be written as rational function in $\nu$. In fact, for the classical discrete orthogonal polynomials, the three-term recurrence relation coefficients $\beta_{n}$ and $\gamma_{n}$ are rational functions in the variable $n$. Using Eq. (56) we get

$$
\Phi(r)=\mathbb{F}_{n}^{(r)}\left(P_{n}^{(r)}(x)\right)=0, \quad \forall r \in \mathbb{N} .
$$

We then conclude that $\Phi(\nu)$ is a rational function with an infinite number of zeros. Therefore, $\Phi(\nu)=0, \forall \nu \in \mathbb{R}_{+}$and $\left(P_{n}^{(\nu)}\right)_{n}$ satisfies Eq. (86).

2. Equation (87) is proved by a straightforward computation using $U_{\nu}$ and $U_{\nu}$ which satisfies Eq. (88). 
3. The functions given in Eq. (90) are represented as products of functions satisfying homogeneous difference equation of order 1 (for $\rho)$ and 2 (for $\mathrm{U}$ and $\mathrm{V})$. These functions, therefore satisfy a difference equation of order $4(=1 \times 2 \times 2)$ which is identical to Eq. (86). Notice that by linear algebra one can deduce the difference equation of the product (90), given the difference equations of the factors, since they have polynomial coefficients. This can be done, e.g. by Maple command "rec*rec" [35] of the gfun package.

We conclude the proof by noticing that the results of the previous theorem can be used to extend Theorem 5 to the generalized co-recursive associated of classical discrete orthogonal polynomials with real order of association as was done for classical continuous in Ref. [9].

\subsection{Solution of Some Second-order Difference Equations}

The factorization pointed out in Eq. (51) can be used to prove the following:

Proposition 3 Two linearly independent solutions of the difference equation

$$
\mathbb{S}_{n}^{(r)}(y)=0,
$$

are

$$
E_{n}^{(r)}(x)=\mathbb{T}_{n}^{(r)}\left(C_{n}^{(r)}(x)\right), \quad F_{n}^{(r)}(x)=\mathbb{T}_{n}^{(r)}\left(D_{n}^{(r)}(x)\right),
$$

where the operators $\mathbb{S}_{n}^{(r)}$ and $\mathbb{T}_{n}^{(r)}$ are given by Eqs. (49) and (50), respectively, and the functions $C_{n}^{(r)}(x)$ and $D_{n}^{(r)}(x)$ given by Eq. (57).

Proposition 4 Two linearly independent solutions of the difference equation

$$
\mathbb{S}_{n}^{(\nu)}(y)=0,
$$

are

$$
E_{n}^{(\nu)}(x)=\mathbb{T}_{n}^{(\nu)}\left(C_{n}^{(\nu)}(x)\right), \quad F_{n}^{(\nu)}(x)=\mathbb{T}_{n}^{(\nu)}\left(D_{n}^{(\nu)}(x)\right),
$$

where the operators $\mathbb{S}_{n}^{(\nu)}$ and $\mathbb{T}_{n}^{(\nu)}$ are given by $E q .(87)$, and the functions $C_{n}^{(\nu)}(x)$ and $D_{n}^{(\nu)}(x)$ given by Eq. (90).

Proof Since the functions $C_{n}^{(r)}$ and $D_{n}^{(r)}$ are solutions of equation $\mathbb{F}_{n}^{(r)}(y)=0$ (see Theorem 2$)$, we use the factorization given by Eq. (51) and get

$$
\mathbb{S}_{n}^{(r)}\left(\mathbb{T}_{n}^{(r)}(y)\right)=X\left(\sigma, \tau, P_{r-1}, \lambda_{r-1}\right) \mathbb{F}_{n}^{(r)}(y)=0
$$

for $y \in\left\{C_{n}^{(r)}, D_{n}^{(r)}\right\}$. We therefore, conclude that the functions $E_{n}^{(r)}$ and $F_{n}^{(r)}$ satisfy $\mathbb{S}_{n}^{(r)}(y)=0$. The proof of Proposition 4 is similar to the one of Proposition 3 by using Theorem 7.

Remark 5 The previous propositions give solutions to families of second-order difference equations. In particular, Proposition 3 solves a family of second-order difference equations with polynomial coefficients. The two previous propositions, given for the associated classical discrete orthogonal polynomials can be used to solve the difference equation $\mathbb{S}_{n}(y)=0$ where $\mathbb{S}_{n}$ is the left factor of the factored form of the fourth-order difference 
operator $\mathbb{F}_{n}\left(\mathbb{F}_{n}=\mathbb{S}_{n} \mathbb{T}_{n}\right)$ for other modifications of classical discrete orthogonal polynomials (see "Perturbation of recurrence coefficients" section).

\subsection{Extension of Results to Semi-classical Cases}

The proof of Theorem 1, which is the starting point of this paper, uses merely the secondorder difference equation (5) and the relation (32). Now we suppose that the family $\left(P_{n}\right)_{n}$ is semi-classical discrete $[7,14,22,25,26,34]$. This implies that $\left(P_{n}\right)_{n}$ is orthogonal satisfying a second-order difference equation of the form

$$
\bar{M}_{n}(y(x))=I_{2}(x, n) y(x+2)+I_{1}(x, n) y(x+1)+I_{0}(x, n) y(x)=0,
$$

where the coefficients $I_{i}(x, n)$ are polynomials in $x$ of degree not depending on $n$.

For semi-classical orthogonal polynomials an equation of type (32) is known and can be stated as $[7,10]$

$$
\tilde{\mathbb{M}}_{n}\left(P_{n-1}^{(1)}(x)\right)=a_{1}(x) P_{n}(x+1)+a_{0}(x) P_{n}(x),
$$

where $a_{i}$ are polynomials and $\tilde{M}_{n}$ a second-order linear difference operator with polynomial coefficients. Use of the two previous equations leads to the following extension.

THEOREM 8 Given $\left(P_{n}\right)_{n}$ a sequence of semi-classical orthogonal polynomials satisfying Eq. (91) and $\left(\bar{P}_{n}\right)_{n}$ a family of orthogonal polynomials obtained by modifying $\left(P_{n}\right)_{n}$ and satisfying

$$
\bar{P}_{n}(x)=A_{n}(x) P_{n+k-1}^{(l)}+B_{n}(x) P_{n+k}, \quad n \geq k^{\prime},
$$

where $A_{n}$ and $B_{n}$ are polynomials of degree not depending on $n$, and $k, k^{\prime} \in \mathbb{N}$, we have the following:

1. The orthogonal polynomials $\left(\bar{P}_{n}\right)_{n \geq k^{\prime}}$ satisfy a common fourth-order linear difference equation

$$
\begin{aligned}
\overline{\mathbb{F}}_{n}(y(x))= & K_{4}(x, n) y(x+4)+K_{3}(x, n) y(x+3)+K_{2}(x, n) y(x+2)+K_{l}(x, n) y(x+1) \\
& +K_{0}(x, n) y(x) \\
= & 0
\end{aligned}
$$

where the coefficients $K_{i}$ are polynomials in $x$, with degree not depending on $n$.

2. The operator $\mathbb{\mathbb { F }}_{n}$ can be factored as product of two second-order linear difference operators

$$
\overline{\mathbb{F}}_{n}=\overline{\mathbb{S}}_{n} \overline{\mathbb{T}}_{n}
$$

where the coefficients of $\overline{\mathbb{S}}_{n}$ and $\overline{\mathbb{T}}_{n}$ are polynomials of degree not depending on $n$.

The proof is similar to the one of Theorem 1 but with Eqs. (91) and (92) playing the role of Eqs. (5) and (32), respectively.

The previous theorem covers many modifications of the recurrence coefficients of the semi-classical discrete orthogonal polynomials, and in particular, the modifications such as 
the associated, the general co-recursive, the general co-dilated, the general co-recursive associated and the general co-modified semi-classical discrete orthogonal polynomials.

When the orthogonal polynomial sequence $\left(P_{n}\right)_{n}$ is semi-classical discrete, it is difficult in general to represent the coefficients of the difference operators, $\overline{\mathbb{M}}_{n}, \tilde{\mathbb{M}}_{n}, \overline{\mathbb{F}}_{n}, \overline{\mathbb{S}}_{n}$ and $\overline{\mathbb{T}}_{n}$ in terms of polynomials $\phi$ and $\psi$, the coefficients of the functional equation (see Refs. $[7,13,22,34])$ satisfied by the regular functional with respect to which $\left(\bar{P}_{n}\right)_{n}$ is orthogonal.

However, for particular cases (for example if the degrees of polynomials $\phi$ and $\psi$ are small), it is possible after huge computations to give the coefficients of the difference operators $\overline{\mathbb{M}}_{n}, \tilde{\mathbb{M}}_{n}, \overline{\mathbb{F}}_{n}, \overline{\mathbb{S}}_{n}$ and $\overline{\mathbb{T}}_{n}$ explicitly, and therefore look for functions annihilating these difference operators.

\section{Acknowledgements}

We are very greateful to the Alexander von Humboldt Foundation, whose grant (Fellowship No IV KAM 1070235), awarded to the first author Mama Foupouagnigni, was decisive for the realization of this work.

\section{References}

[1] N. M. Atakishiyev, A. Ronveaux and K. B. Wolf, Difference equation for the associated polynomials on the linear lattice, Zt. Teor. Mat. Fiz., 106 (1996), 76-83.

[2] T. S. Chihara, On co-recursive orthogonal polynomials, Proc. Am. Math. Soc., 8 (1957), 899-905.

[3] T. S. Chihara, Introduction to Orthogonal Polynomials, Gordon and Breach, New York, 1978.

[4] J. Dini, Sur les formes linéaires et polynômes orthogonaux de Lagurre-Hahn, Thèse de Doctorat, Université Pierre et Marie Curie, Paris VI, 1988.

[5] J. Dini, P. Maroni and A. Ronveaux, Sur une perturbation de la récurrence vérifée par une suite de polynômes orthogonaux, Portugaliae Math., 6(3) (1989), 269-282.

[6] J. Favard, Sur les polynômes de Tchebicheff, C. R. Acad. Sci. Paris, 200 (1935), 2052-2053.

[7] M. Foupouagnigni, Laguerre-Hahn Orthogonal Polynomials with Respect to the Hahn Operator: Fourth-order Difference Equation for the $r$ th Associated and the Laguerre-Freud Equations for the Recurrence Coefficients, Ph.D. Thesis, Université Nationale du Bénin, Bénin, 1998.

[8] M. Foupouagnigni, W. Koepf and A. Ronveaux, Fourth-order difference equation for the associated classical discrete orthogonal polynomials, J. Comp. Appl. Math., 92 (1998), 103-108.

[9] M. Foupouagnigni, W. Koepf and A. Ronveaux, Factorization of fourth-order differential equations for perturbed classical orthogonal polynomials, 2002, submitted for publication.

[10] M. Foupouagnigni, M.N. Hounkonnou and A. Ronveaux, The fourth-order difference equation satisfied by the associated orthogonal polynomials of the $\Delta$-Laguerre-Hahn class, J. Symbolic Comp., 28 (1999), 801-818.

[11] M. Foupouagnigni and F. Marcellán, Characterization of the $D_{\omega}$ Laguerre-Hahn functionals, J. Diff. Eq. Appl., 8 (2002), 689-717.

[12] A. G. Garcia, F. Marcellán and L. Salto, A distributional study of discrete classical orthogonal polynomials, J. Comp. Appl. Math., 57 (1995), 147-162.

[13] M. Guerfi, Les polynômes de Laguerre-Hahn affines discrets, Thése de 3 éme cycle, Université Pierre et Marie Curie, Paris VI (1988).

[14] E. Hendriksen and H. van Rossum, Semi-classical orthogonal polynomials, Polynômes Orthogonaux et Applications, Lect. Notes in Math., Vol. 1171, 1985, pp. 355-361.

[15] W. Koepf, Hypergeometric Summation, Vieweg, Braunschweg/Wiesbaden, 1998.

[16] W. Koepf and D. Schmersau, Representations of orthogonal polynomials, J. Comp. Appl. Math., 90 (1998), 57-94.

[17] R. Koekoek and R. Swarttouw, The Askey-scheme of hypergeometric orthogonal polynomials and its $q$-analogue, Faculty of Information Technology and Systems, Delft University of Technology, Report No. 98-17, 1998.

[18] P. Lesky, Über Polynomlösungen von Differentialgleichungen und Differenzengleichungen zweiter Ordnung, Anzeiger der Österreichischen Akademie der Wissenschaften, Math.-Naturwiss. Klasse, 121 (1985), 29-33.

[19] J. Letessier, A. Ronveaux and G. Valent, Fourth-order difference equation for the associated Meixner and Charlier polynomials, J. Comp. Appl. Math., 71(2) (1996), 331-341.

[20] J. Letessier, Fourth-order difference equation for the co-recursive associated Meixner polynomials, J. Comp. Appl. Math., 103 (1999), 323-335. 
[21] A. Magnus, Riccati Acceleration of Jacobi Continued Fractions and Laguerre-Hahn Orthogonal Polynomials, Lect. Notes Math., Vol. 1071, Springer-Verlag, Berlin, 1984, pp. 213-230.

[22] F. Marcellán and L. Salto, Discrete semiclassical orthogonal polynomials, J. Diff. Eq., 4 (1998), $463-496$.

[23] F. Marcellán, J. S. Dehesa and A. Ronveaux, On orthogonal polynomials with perturbed recurrence relations, J. Comp. Appl. Math., 30(2) (1990), 203-212.

[24] F. Marcellán and E. Prianes, Perturbations of Laguerre-Hahn functionals, J. Comp. Appl. Math., 105 (1999), $109-128$.

[25] P. Maroni, Prolégomènes á l'étude des polynômes orthogonaux semi-classiques, Annali di Mat. Pura ed Appl., 4 (1987), 165-184.

[26] P. Maroni, Une théorie algébrique des polynômes orthogonaux: applications aux polynômes orthogonaux semiclassiques, In: C. Brezinski, et al. ed, Orthogonal Polynomials and Applications, Annals on Computing and Appl. Math., Vol. 9, J.C. Baltzer AG, Bassel, 1991, pp. 98-130.

[27] M. B. Monagan, K. O. Geddes, K. M. Heal, G. Labahn, S. M. Vorkoetter, J. McCarron and P. DeMarco, Maple 8, Waterloo Maple, Inc, 2002.

[28] A. F. Nikiforov and V. B. Uvarov, Special functions of Mathematical Physics, Birkhäuser, Basel/Boston, 1988.

[29] A. F. Nikiforov, S. K. Suslov and V. B. Uvarov, Classical Orthogonal Polynomials of a Discrete Variable, Springer, Berlin, 1991

[30] A. Ronveaux, Discrete semi-classical orthogonal polynomials: generalized Meixner, J. Approx. Theory, 46(4) (1986), 403-407.

[31] A. Ronveaux, S. Belmehdi, J. Dini and P. Maroni, Fourth-order difference equation for the co-modified semiclassical orthogonal polynomials, J. Comp. Appl. Math., 29(2) (1990), 225-231.

[32] A. Ronveaux, E. Godoy, A. Zarzo and I. Area, Fourth-order difference equation for the first associated of classical discrete orthogonal polynomials, J. Comp. Appl. Math., 59 (1998), 47-52.

[33] A. Ronveaux and W. Van Assche, Upward extension of the Jacobi matrix for orthogonal polynomials, J. Approx. Theory, 86(3) (1996), 335-357.

[34] D. Salto, Polinomios $D_{\omega}$ semi-clássicos, Tesis Doctoral, Universidad de Alcalá de Henares, Spain 1995.

[35] B. Salvy and P. Zimmerman, GFUN: A Maple package for the manipulation of generating and holonomic functions in one variable, ACM Trans. Math. Software, 20 (1994), 163-177.

[36] A. Zarzo, A. Ronveaux and E. Godoy, Fourth-order differential equation satisfied by the associated of any order of all classical orthogonal polynomials. A study of their distribution of zeros, J. Comp. Appl. Math., 49 (1993), 349-359. 